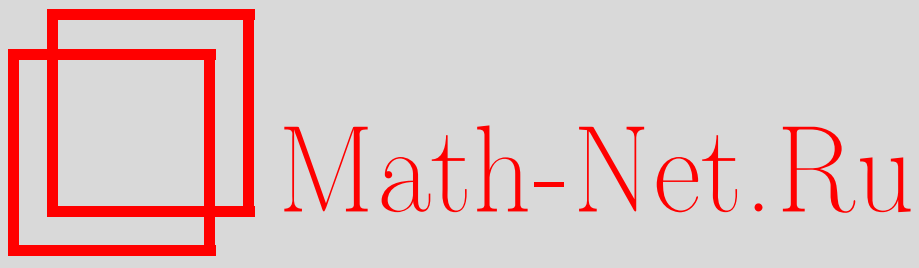

С. Н. Туманов, А. А. Шкаликов, О предельном поведении спектра модельной задачи для уравнения Орра-Зоммерфельда с профилем Пуазейля, Изв. РАН. Сер. матем., 2002, том 66, выпуск 4, 177-204

DOI: https://doi.org/10.4213/im399

Использование Общероссийского математического портала Math-Net.Ru подразумевает, что вы прочитали и согласны с пользовательским соглашением

http: //www. mathnet.ru/rus/agreement

Параметры загрузки:

IP : 54.147 .182 .235

26 апреля 2023 г., 13:05:55 


\author{
С.Н. Туманов, А.А. Шкаликов
}

\title{
О предельном поведении спектра модельной задачи для уравнения Орра-Зоммерфельда с профилем Пуазейля
}

\begin{abstract}
Изучается задача о предельном поведении спектра оператора $L(\varepsilon) y=i \varepsilon y^{\prime \prime}+$ $x^{2} y$ с краевыми условиями Дирихле на конечном отрезке, когда положительный параметр $\varepsilon$ стремится к нулю. Доказано, что спектр концентрируется вдоль трех кривых в комплексной плоскости, соединяющих точку-узел $\lambda_{0}$, лежащую в числовом образе оператора с точками 0,1 и $-i \infty$. Найдены равномерные по $\varepsilon$ квазиклассические формулы распределения собственных значений вдоль этих трех кривых.

Библиограффия: 7 наименований.
\end{abstract}

\section{§1. Введение}

В настоящей работе изучается семейство дифференщиальных операторов

$$
L(\varepsilon) y=i \varepsilon y^{\prime \prime}+x^{2} y, \quad \varepsilon>0,
$$

на отрезке $[-1,1]$ с краевыми условиями

$$
y(-1)=y(1)=0 .
$$

Цель работы - показать, что при малых значениях $\varepsilon$ собственные значения операторов $L(\varepsilon)$ конщентрируются около некоторых кривых в комплексной плоскости, вид которых укажем явно. Найдем асимптотические формулы распределения собственных значений, равномерные по $\varepsilon$, и укажем функции распределения собственных значений вдоль критических линий.

Подобная задача, когда вместо функции $q(x)=x^{2}$ в (1.1) участвует функция $q(x)=x$, изучена в работе [1], где предельное множество, вдоль которого концентрируются собственные значения, названо спектральным галстуком. Граф критических линий в изучаемой задаче для $q(x)=x^{2}$ по форме также напоминает галстук, но только несимметричный. Будем называть его предельным спектральнылм графом.

В [1] отмечено, что форма предельного спектрального граф̆а для профиля $q(x)$ определяется топологией граффов Стокса функции $i(q(z)-\lambda)$. В общем случае определение критических линий концентрации собственных значений - сложная задача. Нелегкой эта задача является даже для конкретной функции $q(x)=x^{2}$.

Работа выполнена при финансовой поддержке Российского фонда фундаментальных исследований (гранты № 01-01-00691, № 00-15-96100).

(C) С.Н. ТУМАнов, А.А. ШКАЛИКОВ, 2002 
Отметим, что рассматриваемая задача является моделью для известного в гидродинамике уравнения Орра-Зоммерфельда с профилем Пуазейля. Некоторые аргументы, поясняющие, что задача (1.1), (1.2) может служить моделью для уравнения Орра-Зоммерфельда, приведены в [2]. При этом роль малого параметра играет число $\varepsilon=1 / R$, где $R$ - число Рейнольдса, которое обратно пропорционально вязкости жидкости. Таким образом, малым значениям параметра $\varepsilon$ соответствует уравнение движения жидкости, близкой к идеальной.

\section{§2. Общие замечания о спектре}

Пусть $L=L(\varepsilon)$ - оператор, порожденный дифференциальным выражением

$$
L y=i \varepsilon y^{\prime \prime}+x^{2} y
$$

и краевыми условиями

$$
y(-1)=y(1)=0
$$

здесь $\varepsilon-$ мальй положительный параметр.

ПРЕДЛОЖЕНИЕ 2.1. Спектр оператора $L(\varepsilon)$ лежит в полуполосе

$$
\Pi_{\varepsilon}=\left\{\xi \in \mathbb{C} \mid 0<\operatorname{Re} \xi<1, \operatorname{Im} \xi<-\frac{\varepsilon \pi^{2}}{4}\right\}
$$

ДокАЗАТЕЛЬСтво. Известно, что спектр оператора $L$ заключен в его числовом образе, т. е. во множестве значений квадратичной формы $(L y, y)$, когда $y$ пробегает единичную сферу. В нашем случае имеем

$$
(L y, y)=-i \varepsilon\left(y^{\prime}, y^{\prime}\right)+\left(x^{2} y, y\right) .
$$

Первое собственное значение оператора $L_{0} y=-y^{\prime \prime}$ с рассматриваемыми краевыми условиями Дирихле равно $\pi^{2} / 4$, поэтому

$$
\frac{\pi^{2}}{4} \int_{-1}^{1}|y(x)|^{2} d x \leqslant \int_{-1}^{1}\left|y^{\prime}(x)\right|^{2} d x
$$

Отсюда получается сформулированное предложение.

ПрЕДЛОЖЕнИЕ 2.2. При любом $\varepsilon>0$ спектр L cocтоит из последовательности собственных значений $\left\{\lambda_{n}\right\}$, для которьх существует предел вещественных частей:

$$
\lim _{n \rightarrow \infty} \operatorname{Re} \lambda_{n}=\frac{1}{3} .
$$

Более того, справедлива асимптотика

$$
\lambda_{n}=-\frac{\pi^{2} i}{4 \varepsilon} n^{2}+\frac{1}{3}+O\left(\frac{1}{n^{2}}\right)
$$


ДокАЗАТЕЛЬСТво. Проведем замену $x=2 t / \pi-1, \mu=4 i \lambda /\left(\pi^{2} \varepsilon\right)$. Тогда краевая задача (1.1), (1.2) примет вид

$$
-z^{\prime \prime}+\frac{i}{\varepsilon} \frac{4}{\pi^{2}}\left(\frac{2 t}{\pi}-1\right)^{2} z, \quad z(0)=z(\pi)=0 .
$$

Известно [3], что собственные значения задачи имеют асимптотику:

$$
\sqrt{\mu_{n}}=n+\frac{b_{0}}{2 n}+O\left(\frac{1}{n^{2}}\right)
$$

где

$$
b_{0}=\frac{1}{\pi} \int_{0}^{\pi} g(t) d t, \quad g(t)=\frac{4 i}{\pi^{2} \varepsilon}\left(\frac{2 t}{\pi}-1\right)^{2} .
$$

Делая обратную замену, получим требуемую формулу. Предложение доказано.

Обрашаем внимание на то, что остаточный член в формуле (2.2) имеет оценку $\left|O\left(n^{-2}\right)\right| \leqslant C(\varepsilon) n^{-2}$, где постоянная $C(\varepsilon)$ зависит от $\varepsilon$. Однако далее будет установлена равномерная оценка по $\varepsilon$ и по $n$.

Далее через П обозначаем полуполосу (2.1) при $\varepsilon=0$. Из леммы 2.1 следует, что предельное множество концентрации спектра оператора $L(\varepsilon)$ при $\varepsilon \rightarrow 0$ лежит в П.

\section{§3. Граф Стокса}

Напомним некоторые понятия, используемые в теории графов Стокса целых функций (подробное изложение этой теории см. в [5]).

ОПРЕДЕЛЕНИЕ 3.1. Нули целой функции $q$ называются точками поворота. Точка поворота называется простой, если она является простым нулем функции $q$.

Введем многозначную функцию двух переменных

$$
S(a, z)=\int_{a}^{z} \sqrt{q(\zeta)} d \zeta
$$

ОПРЕДЕЛЕнИЕ 3.2. Пусть $a$ - точка поворота целой функции $q$. Аналитические кривые, определенные уравнением $\operatorname{Re} S(a, z)=0$, называются линиями Cтокса, выходящими из точки поворота а. Аналитические кривые, определяемые уравнением $\operatorname{Im} S(a, z)=0$, называются сопряженнымми линиями Стокса, выходящими из точки поворота а.

ОПРЕДЕЛЕНИЕ 3.3. Объединение всех линий Стокса называется графом Cmoкca. Связная компонента графа Стокса называется комплексом Cтокса.

ОПРЕДЕЛЕниЕ 3.4. Максимальные области однолистности функции $S$, ограниченные линиями Стокса, будем называть каноническими областями.

Легко заметить, что граница канонической области содержит, по меньшей мере, одну точку поворота, а сама область - единственную линию Стокса, выходящую из этой точки. 
ОПРЕДЕЛЕНИЕ 3.5 . Тройку $\left(D_{j}, l_{j}, a_{j}\right)$, где $a_{j}$ - точка поворота, $l_{j}-$ линия Стокса, выходяшая из этой точки, $D_{j}-$ каноническая область, содержащая $l_{j}$, будем называть канонической.

ОПРЕДЕЛЕНИЕ 3.6. Пусть $\left(D_{j}, l_{j}, a_{j}\right)$ - каноническая тройка. Ветвь функции $S\left(a_{j}, z\right)$, для которой $\operatorname{Im} S\left(a_{j}, l_{j}\right)=[0 ;+\infty)$, будем называть канонической ветвью, соответствующей данной тройке.

В случае зависимости функции $q$ от некоторого параметра $\lambda$ получаем семейство графов Стокса $\Phi(\lambda)$.

ОПРЕДЕЛЕНИЕ 3.7. Графы $\Phi\left(\lambda_{1}\right), \Phi\left(\lambda_{2}\right)$ әквивалентны, если существует гомеоморфизм

$$
\phi: \Phi\left(\lambda_{1}\right) \rightarrow \Phi\left(\lambda_{2}\right)
$$

при котором точки поворота переходят в точки поворота.

ОПРЕДЕЛЕНИЕ 3.8 . Точка $\lambda_{0}$ назьвается допустимой, если существует окрестность $O\left(\lambda_{0}\right)$ такая, что для всех точек $\lambda$ этой окрестности графы $\Phi\left(\lambda_{0}\right)$ и $\Phi(\lambda)$ эквивалентны. В противном случае точка $\lambda_{0}$ называется недопустимой.

Пусть

$$
q(z, \lambda)=a_{0}(\lambda)\left(z-b_{1}(\lambda)\right)^{n_{1}} \ldots\left(z-b_{k}(\lambda)\right)^{n_{k}}, \quad n_{j} \in \mathbb{N},
$$

где $b_{j}$ - алгебраические функции, $a_{0} \not \equiv 0$ - полином.

Положим

$$
\Sigma(a, b, \lambda)=\int_{a}^{b} \sqrt{q(\zeta, \lambda)} d \zeta
$$

Пусть $I_{1}$ - нули $a_{0}(\lambda), I_{2}$ - множество всех $\lambda$ таких, что $b_{j}(\lambda)=b_{l}(\lambda)$ при $j \neq l$, $I_{3}$ - множество всех $\lambda$ таких, что $\operatorname{Re} \Sigma\left(b_{j}(\lambda), b_{l}(\lambda), \lambda\right)=0$ при некоторых $j \neq l$.

Справедлива следуюшая лемма (см. $[5, \S 3$, п. 5$])$.

Лемма 3.1. Множество всех недопустимых точек есть

$$
I=I_{1} \cup I_{2} \cup I_{3} .
$$

В этом параграфе изучим топологию графа Стокса функции $q(z, \lambda)=i\left(z^{2}-\lambda\right)$ при $\lambda \in \Pi$ П, где

$$
\Pi=\{\xi \in \mathbb{C} \mid 0<\operatorname{Re} \xi<1, \operatorname{Im} \xi<0\} .
$$

ЛЕмма 3.2. Для функиии $q(z, \lambda)=i\left(z^{2}-\lambda\right)$ имеем

$$
I_{1}=\varnothing, \quad I_{2}=\{0\}, \quad I_{3}=\left\{\lambda \mid \arg \lambda=-\frac{\pi}{4}\right\}
$$

т.е. мнохсество недопустимых точек в П совпадает с лучом $\arg \lambda=-\pi / 4$. 
ДоКАЗАТЕЛЬСтво. Для множеств $I_{1}$ и $I_{2}$ утверждение леммы очевидно. Согласно определению множество точек $\lambda \in I_{3}$ определяется уравнением

$$
\operatorname{Re}\left(e^{\frac{\pi}{4} i} \int_{-\sqrt{\lambda}}^{\sqrt{\lambda}} \sqrt{\zeta^{2}-\lambda} d \zeta\right)=0
$$

После замены $\xi=\zeta / \sqrt{\lambda}$ это уравнение приводится к виду

$$
\operatorname{Re}\left(\lambda e^{\frac{\pi}{4} i} \int_{-1}^{1} \sqrt{\xi^{2}-1} d \xi\right)=0
$$

что эквивалентно уравнению $\operatorname{Re}\left(\lambda e^{\frac{\pi}{4} i} i\right)=0$. Лемма доказана.

Введем ветвь $\sqrt{\lambda}$, которую всюду далее будем называть основной: $\arg \sqrt{\lambda} \epsilon$ $(-\pi / 4,0)$ при $\lambda \in \Pi$. Если не оговорено противное, предполагаем, что $\lambda \in$ П. Из обшей теории графов Стокса [5] следует, что граф Стокса рассматриваемой функции в зависимости от $\lambda$ может либо быть связным, либо содержать два комплекса Стокса. В последнем случае каждый из комплексов состоит из трех линий Стокса, выходящих из точки поворота под углами $2 \pi / 3$ по отношению друг к другу. Линии Стокса каждого комплекса можно условно разделить на внутренние (по две на комплекс) и внешние (по одной на комплекс). Внутренние линии характеризуются асимптотическим стремлением к линиям Стокса парного комплекса (см. рис. 1).

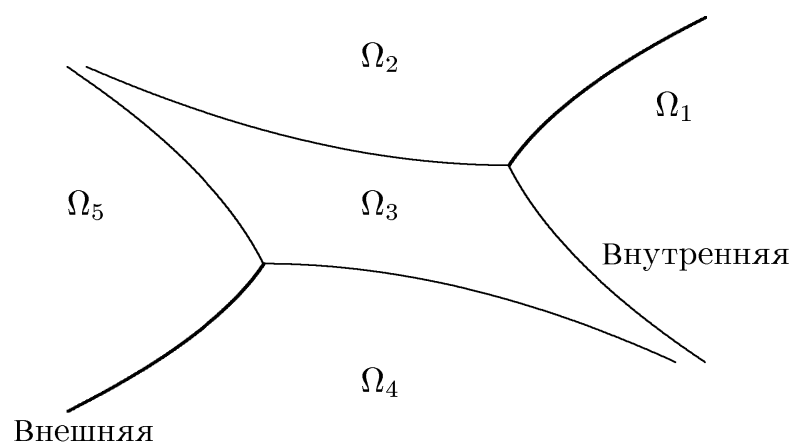

Рис. 1. Внешние и внутренние линии графа Стокса

В изучаемом случае точками поворота являются $\pm \sqrt{\lambda}$. Уравнение для графа Стокса будет выглядеть следуюшим образом:

$$
\operatorname{Re}\left(e^{\frac{\pi}{4} i} \int_{ \pm \sqrt{\lambda}}^{z} \sqrt{\zeta^{2}-\lambda} d \zeta\right)=0
$$

После замены переменных в интеграле и сокращения на $|\lambda|$ это уравнение можно переписать в виде

$$
\operatorname{Re}\left(e^{\left(\frac{\pi}{4}+\psi\right) i} \int_{1}^{ \pm z / \sqrt{\lambda}} \sqrt{\zeta^{2}-1} d \zeta\right)=0, \quad \psi=\arg \lambda \in(-\pi / 2,0) .
$$

Далее через $\Gamma=\Gamma(\lambda)$ обозначим граф Стокса функции $Q(z \lambda)=i\left(z^{2}-\lambda\right)$. 
ЛЕмма 3.3. Граф Стокса Г обладает свойством чентральной симметрии относительно точки 0.

ДокАЗАТЕЛЬСтво. Утверждение следует из четности функции $q=i\left(z^{2}-\lambda\right)$. Таким образом, если граф Г не связный, а состоит из двух комплексов, то достаточно изучать топологию одного комплекса Стокса.

Лемма 3.4. Граф Г $\lambda($ связный тогда и только тогда, когда $\psi=\arg \lambda=$ $-\pi / 4$.

ДокаЗАТЕЛЬСтво. Связность графа Стокса равносильна принадлежности точек $\pm \sqrt{\lambda}$ одному комплексу, из чего согласно $(3.2)$ получим

$$
\operatorname{Re}\left(e^{\left(\frac{\pi}{4}+\psi\right) i} \int_{-1}^{1} \sqrt{\zeta^{2}-1} d \zeta\right)=0 .
$$

Поскольку

$$
\int_{-1}^{1} \sqrt{\zeta^{2}-1}=i \frac{\pi}{2}
$$

то последнее уравнение выполняется в том и только том случае, когда $\psi=-\pi / 4$. Лемма доказана.

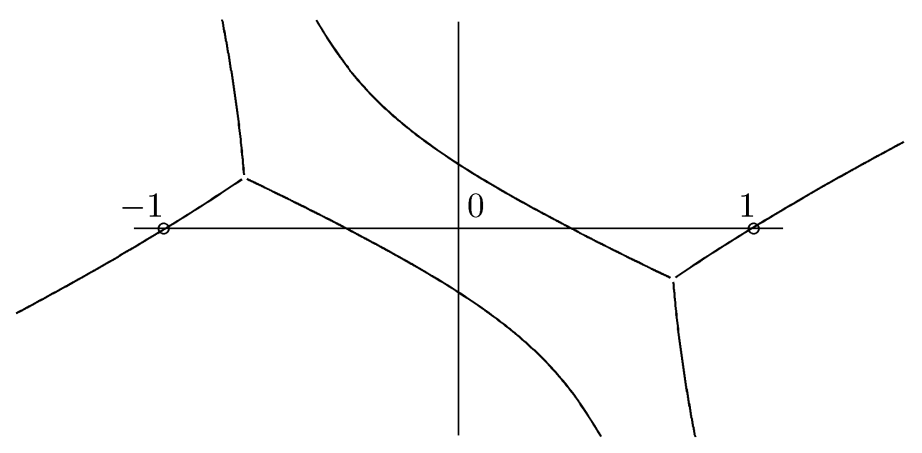

Рис. 2. Линии Стокса в случае $\arg \lambda \in(-\pi / 4,0)($ для $\lambda=1 / 2-i / 4)$

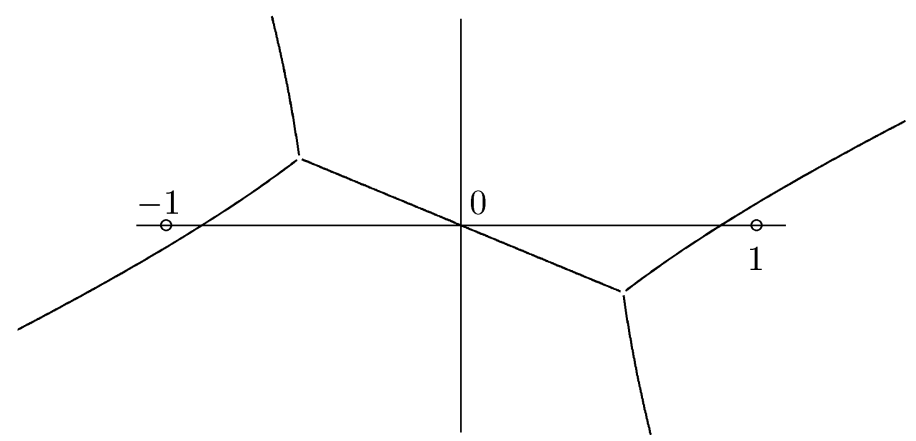

Рис. 3. Линии Стокса в случае $\arg \lambda=-\pi / 4$ (для $\lambda=1 / 4-i / 4)$ 


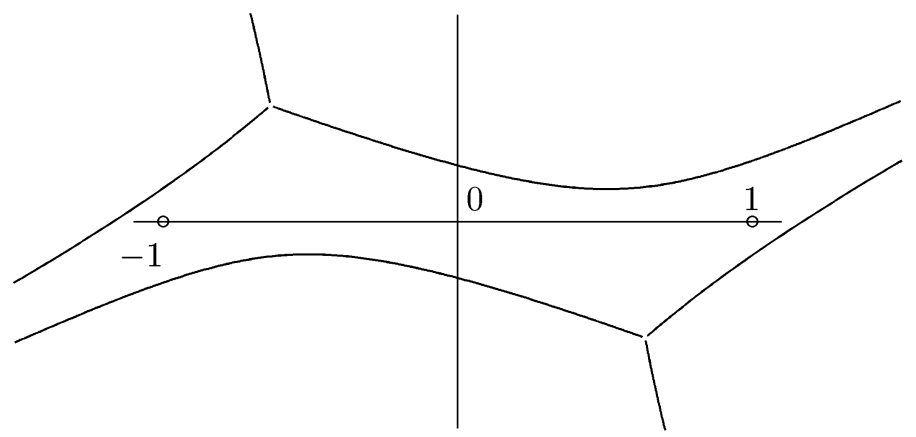

Рис. 4. Линии Стокса в случае $\arg \lambda \in(-\pi / 2,-\pi / 4)$ (для $\lambda=1 / 4-i / 2)$

ЗАмЕчАниЕ 3.1. В дальнейшем важно понимать, какие области являются каноническими для рассматриваемой функции $q$. Рассмотрим два случая.

1) $\arg \lambda \neq-\pi / 4$. Тогдаграф $\Gamma(\lambda)$ состоит из двух комплексов Стокса (см. рис. 1$)$, которые разбивают плоскость на области двух типов: типа полосы (область, ограниченная внутренними линиями Стокса, на рисунке она обозначена $\Omega_{3}$ ) и типа полуплоскости (все остальные области). В этом случае канонические области могут быть двух типов:

а) области, состоящие из двух областей типа полуплоскости и содержащие одну внешнюю линию Стокса (например, $\Omega_{1} \cup \Omega_{2}$ );

b) области, содержащие область типа полосы и две примыкающие к ней области типа полуплоскости, отвечающие различным комплексам Стокса (например, $\Omega_{1} \cup$ $\Omega_{3} \cup \Omega_{4}, \Omega_{1} \cup \Omega_{3} \cup \Omega_{5}$; см. рис. 1).

$2) \arg \lambda=-\pi / 4$. Тогда граф Стокса состоит из одного комплекса, разбивающего плоскость на четыре области типа полуплоскости. В этом случае канонические области состоят из любых двух соседних областей типа полуплоскости.

Лемма 3.5. Граф Г связный тогда и только тогда, когда $0 \in \Gamma$.

ДоказАТЕльство. Достаточность очевидна, докажем необходимость. Из предыдущей леммы имеем $\psi=-\pi / 4$. Подставляя в (3.2) значение аргумента, получаем уравнение, которому удовлетворяет точка 0 . Лемма доказана.

ЛЕмма 3.6. Если граф Г состоит из двух комплексов, то точка 0 лежит внутри области, ограниченной внутренними линиями Стокса обоих комплексов.

ДокАЗАтЕльство. Это утверждение вытекает из симметрии граффа Г относительно точки 0.

Далее мы исследуем, какие линии Стокса графа $\Gamma(\lambda)$ пересекают вещественную ось в зависимости от параметра $\lambda \in \Pi$ (см. рис. 2-4).

ЛЕмма 3.7. Если $\arg \lambda=\psi \in(-\pi / 2,-\pi / 4)$, то граф $\Gamma(\lambda)$ состоит из двух комплексов. При этом вещественную положительную полуось пересекает не более одной линии Стокса каждого комплекса. Такое же утверждение справедливо и для отрицательной полуоси. 


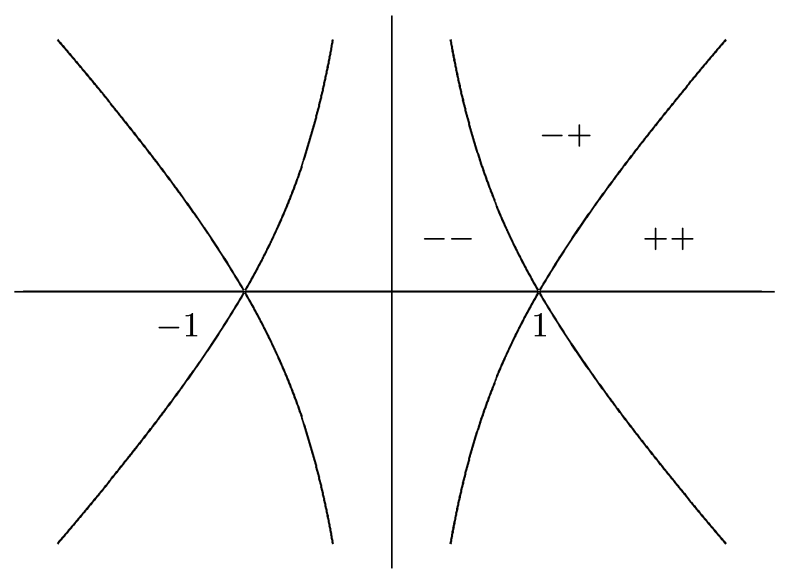

Рис. 5. Линии и сопряженные линии Стокса функции $p(z)=z^{2}-1$

ДокАЗАТЕЛЬСТво. Рассмотрим сначала линии Стокса и сопряженные линии Стокса функции $p(z)=z^{2}-1$ (см. рис. 5). Первые задаются уравнением

$$
\operatorname{Re} \int_{ \pm 1}^{z} \sqrt{\zeta^{2}-1} d \zeta=0
$$

а вторые - уравнением

$$
\operatorname{Im} \int_{ \pm 1}^{z} \sqrt{\zeta^{2}-1} d \zeta=0
$$

Эти кривые разбивают первую четверть на три области, при переходе через гранищы которых меняются знаки либо вешественной, либо мнимой части интеграла $\int_{1}^{z} \sqrt{\zeta^{2}-1} d \zeta$. Рассмотрим ветвь функции $y=\sqrt{\zeta^{2}-1}$, принимающую положительные значения при $\zeta>1$.

Пусть $r \rightarrow+\infty, \varepsilon \rightarrow+0$. Тогда

$$
\int_{1}^{r+i \varepsilon} \sqrt{\zeta^{2}-1} d \zeta=\int_{1}^{r} \sqrt{\zeta^{2}-1} d \zeta+\int_{r}^{r+i \varepsilon} \sqrt{\zeta^{2}-1} d \zeta .
$$

Очевидно, что мнимая и действительная части интеграла положительны. На рис. 5 это отражается знаками ++ , проставленными в соответствующей области. На том же рисунке парами знаков обозначены две оставшиеся области. Первый знак соответствует знаку вешественной части интеграла, второй - знаку мнимой.

Предположим, что найдутся две линии Стокса $l_{1}$ и $l_{2}$ графа $\Gamma(\lambda)$, отвечающего функции $q=i\left(z^{2}-\lambda\right)$, пересекающие вещественную положительную полуось. Пусть $z_{1}$ и $z_{2}-$ точки пересечения. Имеем равенство

$$
\operatorname{Re}\left(e^{\left(\frac{\pi}{4}+\psi\right) i} \int_{1}^{z_{1} / \sqrt{\lambda}} \sqrt{\zeta^{2}-1} d \zeta\right)=\operatorname{Re}\left(e^{\left(\frac{\pi}{4}+\psi\right) i} \int_{1}^{z_{2} / \sqrt{\lambda}} \sqrt{\zeta^{2}-1} d \zeta\right)=0 .
$$

Так как линии Стокса различны, то различными будут знаки мнимых частей:

$$
\operatorname{sgn} \operatorname{Im}\left(e^{\left(\frac{\pi}{4}+\psi\right) i} \int_{1}^{z_{1} / \sqrt{\lambda}} \sqrt{\zeta^{2}-1} d \zeta\right)=-\operatorname{sgn} \operatorname{Im}\left(e^{\left(\frac{\pi}{4}+\psi\right) i} \int_{1}^{z_{2} / \sqrt{\lambda}} \sqrt{\zeta^{2}-1} d \zeta\right) .
$$


Таким образом, можно считать, что найдется $\alpha \in \mathbb{R}$ такое, что

$$
\int_{1}^{\eta} \sqrt{\zeta^{2}-1} d \zeta=-\alpha^{2} \int_{1}^{\gamma \eta} \sqrt{\zeta^{2}-1} d \zeta
$$

Здесь $\eta=z_{1} / \sqrt{\lambda}, \gamma \eta=z_{2} / \sqrt{\lambda}, \gamma>0, \operatorname{a} \arg \eta=\arg \gamma \eta \in(\pi / 8, \pi / 4)$. Последнее следует из условия леммы: $\arg \lambda \in(-\pi / 2,-\pi / 4)$.

Обозначим $\theta=\arg \int_{1}^{\eta} \sqrt{\zeta^{2}-1} d \zeta$. Из соотношения

$$
\operatorname{Re}\left(e^{\left(\frac{\pi}{4}+\psi\right) i} e^{\theta i}\left|\int_{1}^{z_{1} / \sqrt{\lambda}} \sqrt{\zeta^{2}-1} d \zeta\right|\right)=0
$$

получим $\cos (\pi / 4+\psi+\theta)=0$, откуда следует $\theta=\pi / 4-\psi+\pi m$. Так как $-\pi / 2<\psi<-\pi / 4$, имеем $\pi / 2+\pi m<\theta<3 \pi / 4+\pi m$. Стало быть,

$$
\operatorname{sgn} \operatorname{Re} \int_{1}^{\eta} \sqrt{\zeta^{2}-1} d \zeta=-\operatorname{sgn} \operatorname{Im} \int_{1}^{\eta} \sqrt{\zeta^{2}-1} d \zeta
$$

У интеграла $\int_{1}^{\gamma \eta} \sqrt{\zeta^{2}-1} d \zeta$ знаки мнимой и вещественной частей тоже различны, следовательно, значения обоих интегралов лежат в области, обозначенной на рис. 5 знаками -+, что противоречит (3.3). Тем самым утверждение для положительной полуоси доказано. Для отрищательной полуоси утверждение справедливо в силу симметрии.

Teоpema 3.1. Пусть $\arg \lambda=\psi \in(-\pi / 2,-\pi / 4)$. Тогда внешние линии Стокса графа $\Gamma(\lambda)$ не пересекаются $c \mathbb{R}$.

ДоКАЗАТЕЛЬСТво. Все рассуждения будут проводиться для одного комплекса Стокса. Так как внутренние линии Стокса одного комплекса асимптотически сближаются с линиями второго комплекса, то хотя бы одна внутренняя линия обязана пересечь вешественную полуось. Допустим, что внешняя линия Стокса тоже пересечет $\mathbb{R}$. Согласно лемме 3.7 она не может пересечь ту же полуось, что и внутренняя линия, пересекаюшая $\mathbb{R}$, т. е. внутренняя и внешняя линии рассматриваемого комплекса пересекают разные вешественные полуоси. Тогда другая внутренняя линия того же комплекса не может пересекать ни $\mathbb{R}^{+}$, ни $\mathbb{R}^{-}$. Теперь, если рассмотреть весь граф Стокса $\Gamma(\lambda)$, состоящий из двух комплексов, мы получаем, что точка 0 не лежит между внутренними линиями Стокса. Это противоречит лемме 3.6. Теорема доказана.

Teорема 3.2. Пусть $\arg \lambda=\psi \in(-\pi / 4,0)$. Тогда внешние линии Стокса графа $\Gamma(\lambda)$ пересекают вещественную ось $\mathbb{R}$.

ДокАЗАТЕЛЬСТво. Пусть $x$ пробегает значения от 0 до $+\infty$. Проследим значения интеграла $\int_{1}^{\eta} \sqrt{\zeta^{2}-1} d \zeta$ при изменении величины $\eta=x / \sqrt{\lambda}$. Здесь число $\lambda$ фиксировано, $\psi=\arg \lambda \in(-\pi / 4,0)$, поэтому $\arg \eta \in(0, \pi / 8)$. Снова рассмотрим линии и сопряженные линии Стокса функции $p=z^{2}-1$ (рис. 5). При росте $x$ значение интеграла последовательно перемешается из третьей четверти во вторую, из второй в первую. Более того, начальное значение интеграла равно $-\pi i / 4$. 
Найдем предельное значение аргумента при $x \rightarrow+\infty$. Обозначим $\theta=\arg \eta$, $r=|\eta|$. Имеем

$$
\begin{aligned}
\int_{1}^{r e^{i \theta}} \sqrt{\zeta^{2}-1} d \zeta & =\int_{1}^{r} \sqrt{\zeta^{2}-1} d \zeta+\int_{r}^{r e^{i \theta}} \sqrt{\zeta^{2}-1} d \zeta \\
\int_{r}^{r e^{i \theta}} \sqrt{\zeta^{2}-1} d \zeta & =i \int_{0}^{\theta}\left(r e^{i \theta}\right)^{2} \sqrt{1-\frac{1}{\left(r e^{i \theta}\right)^{2}}} d \theta \\
& =\frac{r^{2}}{2}\left(e^{2 i \theta}-1\right)-i \frac{\theta}{2}+o(1), \quad r \rightarrow+\infty .
\end{aligned}
$$

Таким образом, при $r \rightarrow+\infty$

$$
\begin{aligned}
& \operatorname{Re} \int_{1}^{r e^{i \theta}} \sqrt{\zeta^{2}-1} d \zeta=\frac{r^{2}}{2} \cos 2 \theta+o\left(r^{2}\right) \\
& \operatorname{Im} \int_{1}^{r e^{i \theta}} \sqrt{\zeta^{2}-1} d \zeta=\frac{r^{2}}{2} \sin 2 \theta+o\left(r^{2}\right)
\end{aligned}
$$

Следовательно,

$$
\lim _{r \rightarrow+\infty} \arg \int_{1}^{r e^{i \theta}} \sqrt{\zeta^{2}-1} d \zeta=2 \theta .
$$

Поскольку $\theta=-\psi / 2$, получаем

$$
\lim _{r \rightarrow+\infty} \arg e^{(\pi / 4+\psi) i} \int_{1}^{r e^{i \theta}} \sqrt{\zeta^{2}-1} d \zeta=\frac{\pi}{4} .
$$

Поэтому при достаточно больших $x>x_{0}$ значения функции

$$
J(\eta)=e^{(\pi / 4+\psi) i} \int_{1}^{r e^{i \theta}} \sqrt{\zeta^{2}-1} d \zeta, \quad \eta=r e^{i \theta}
$$

будут находиться в первой четверти. Так как $\psi \in(-\pi / 4,0)$, то значение $J(0)$ находится в четвертой четверти. Следовательно, найдутся точки $x \in(0,+\infty)$, при которых значения $J(\eta)$ лежат на отрищательной и положительной мнимых осях. Стало быть, как минимум, две линии Стокса одного из комплексов $\Gamma(\lambda)$ пересекут вещественную положительную полуось.

Покажем, что одна из них обязана быть внешней. Рассмотрим отрезок $[0, \sqrt{\lambda}]$. Он имеет единственную общую точку с графом Стокса. Следовательно, если две внутренние линии пересекут вещественную положительную полуось, то и внешняя линия тоже ее пересечет. Теорема доказана.

Далее изучим положение точек \pm 1 относительно графа Стокса $\Gamma(\lambda)$.

ОПРЕДЕЛЕнИЕ 3.9 . Точку $\lambda \in \Pi$ будем называть регулярной, если точки \pm 1 относительно графа Стокса функции $q(z, \lambda)$ лежат в одной канонической области, в противном случае точку $\lambda$ будем называть сингулярной. 
Tеорема 3.3. Все значения $\lambda \in \Pi$, для которых $\arg \lambda=\psi \in(-\pi / 2,-\pi / 4)$, являются регулярными.

ДоКАЗАТЕЛЬСТво. Из леммы 3.4 следует, что граф̆ $\Gamma(\lambda)$ состоит из двух комплексов Стокса, а из теоремы 3.1 следует, что вся вещественная ось лежит в одной канонической области. Теорема доказана.

Tеорема 3.4. Если $\lambda=r e^{i \pi / 4}$, то найдется точка $r=r_{0}$ такая, что при $r>r_{0}$ значения $\lambda$ являются регулярныцми, а при $0<r \leqslant r_{0}-$ сингулярными. При $r=r_{0}$ точки \pm 1 лежат на линиях Стокса.

ДокаЗАТЕЛЬСтво. В случае $\arg \lambda=\psi=-\pi / 4$ уравнение (3.2) для графа Стокса преобразуется к виду

$$
\operatorname{Re}\left(\int_{1}^{z / \sqrt{\lambda}} \sqrt{\zeta^{2}-1} d \zeta\right)=0
$$

Это равенство эквивалентно принадлежности точки $z / \sqrt{\lambda}$ графу Стокса функции $p(\zeta)=\zeta^{2}-1$. При фиксированном $\lambda$ и $z \in \mathbb{R}^{+}$имеем $\arg z / \sqrt{\lambda}=\pi / 8$. Обращаясь к рис. 5 , легко заметить, что найдется единственная точка $z \in \mathbb{R}^{+}$, удовлетворяющая (3.4). Следовательно, найдется единственная точка $\lambda$ на луче $\arg \lambda=-\pi / 4$, которая удовлетворяет (3.4) при фиксированном $z=1$.

Бесконечные линии Стокса, выходящие из точки 1 , лежат правее прямой $\operatorname{Re} z=$ 1 , что следует из того, что эти линии выходят из точки 1 под углами $\pm \pi / 3$ к лучу $[1,+\infty)$ и частная производная не равна нулю:

$$
\partial \operatorname{Re} \int_{1}^{\xi} \sqrt{\zeta^{2}-1} d \zeta / \partial \operatorname{Im} \xi \neq 0
$$

Следовательно, если $z / \sqrt{\lambda}$ лежит на указанных линиях Стокса и $z=1$, то $|1 / \sqrt{\lambda}|>1$, что влечет $r_{0}=|\lambda|<1$. Таким образом показано, что есть одна точка на луче $\arg \lambda=-\pi / 4$, обладающая тем свойством, что граф Стокса $\Gamma(\lambda)$ проходит через точку $z=1$. Поскольку граф симметричен относительно нуля, то он проходит и через точку $z=-1$.

При изменении $r$ граф $\Gamma(\lambda)$ меняется гомотетично, причем с уменьшением $r$ граф̆ сжимается. Следовательно, при малых $r$ точки \pm 1 лежат в разных областях, границей которых являются только бесконечные линии Стокса (см. рис. 3). Поэтому они не содержатся в одной канонической области. При $r>r_{0}$ точки \pm 1 попадают в области, частью границ которых является отрезок $[-\sqrt{\lambda}, \sqrt{\lambda}]$. Поэтому точки \pm 1 лежат в одной канонической области. Теорема доказана.

Теорема 3.5. При $\lambda=r e^{i \psi}, \psi \in(-\pi / 4,0)$, найдется единственная точка $r=r_{0}(\psi)$ такая, что при $r=r_{0}$ точки \pm 1 лежат на линиях Стокса гра$\oint а \Gamma(\lambda)$. Линии Стокса, содержащие \pm 1 , являются внешними. При прочих $r$ значения $\lambda$ регулярны. 
ДоказАТЕЛЬСтво. Точки $\lambda$ из сектора $\arg \lambda=\psi \in(-\pi / 4,0)$, для которых граф̆ $\Gamma(\lambda)$ проходит через точки \pm 1 , определяются множеством

$$
\gamma_{1}=\left\{\lambda \in \Pi \mid-\frac{\pi}{4}<\arg \lambda<0, \operatorname{Re}\left(e^{\frac{\pi}{4} i} \int_{\sqrt{\lambda}}^{1} \sqrt{\zeta^{2}-\lambda} d \zeta=0\right)\right\} .
$$

Покажем сначала, что при фиксированном $\psi$ множество $\gamma_{1}$ содержит не более одной точки. Предположим, что найдутся две точки $\lambda_{1}, \lambda_{2} \in \gamma$ с одинаковым аргументом. Не ограничивая обшности, можно считать, что $1 / \sqrt{\lambda_{2}}=\alpha / \sqrt{\lambda_{1}}$, где $\alpha>1$.

Рассмотрим функцию

$$
\chi(\xi)=\operatorname{Re}\left(e^{i \frac{\pi}{4}} \lambda_{1} \int_{1}^{\xi / \sqrt{\lambda_{1}}} \sqrt{\zeta^{2}-1} d \zeta\right), \quad \xi \in[0, \alpha] .
$$

Вновь воспользуемся представлением интеграла из (3.5) интегралом вида (3.2). Тогда из нашего предположения следует, что $\chi(\xi)=0$ на конщах отрезка $[0, \alpha]$. Но тогда существует $\tau \in(1, \alpha)$ такое, что

$$
\chi^{\prime}(\tau)=\operatorname{Re}\left(e^{i \frac{\pi}{4}} \sqrt{\lambda_{1}} \sqrt{\tau^{2} / \lambda_{1}-1}\right)=\operatorname{Re}\left(e^{i \frac{\pi}{4}} \sqrt{\tau^{2}-\lambda_{1}}\right)=0 .
$$

Но это невозможно в силу положительности функции $\operatorname{Re}\left(e^{i \pi / 4} \sqrt{\xi^{2}-\lambda_{1}}\right)$ при $\lambda_{1} \in$ $\Pi, \xi>1$.

Теперь покажем, что при всех $\psi \in(-\pi / 4,0)$ сушествует точка $\lambda, \arg \lambda=\psi$, удовлетворяюшая условию (3.5), т. е. кривая $\gamma_{1}$ есть функция полярного аргумента $\psi$. В силу эквивалентности уравнений (3.1) и (3.2) уравнение для кривой $\gamma_{1}$ можно переписать в виде

$$
\gamma_{1}=\left\{\lambda \in \Pi \mid-\frac{\pi}{4}<\arg \lambda<0, \operatorname{Re}\left(e^{\left(\frac{\pi}{4}+\psi\right) i} \int_{1}^{1 / \sqrt{\lambda}} \sqrt{\zeta^{2}-1} d \zeta=0\right)\right\} .
$$

Пусть $\lambda \rightarrow 0$ вдоль луча с постоянньм аргументом $\psi$. Повторив рассуждения доказательства теоремы 3.2 , можно получить, что при достаточно малых $|\lambda|<r_{0}$

$$
\operatorname{Re}\left(e^{\left(\frac{\pi}{4}+\psi\right) i} \int_{1}^{1 / \sqrt{\lambda}} \sqrt{\zeta^{2}-1} d \zeta\right)>0
$$

При этом $1 / \sqrt{\lambda}$ лежит в области ++ (см. рис. 5$)$. Далее, несложно понять, что

$$
\operatorname{Re}\left(e^{\left(\frac{\pi}{4}+\psi\right) i} \int_{1}^{1 / \sqrt{\lambda}} \sqrt{\zeta^{2}-1} d \zeta\right)<0
$$

когда $1 / \sqrt{\lambda}$ находится в области -+. Следовательно, рассматриваемое выражение аннулируется в области ++ или на ее гранище. Повторяя рассуждения из теоремы 3.4, получаем, что соответствуюшее значение $\lambda$ по модулю меньше 1 . Следовательно, кривая $\gamma_{1}-$ функция аргумента $\psi=\arg \lambda \in(-\pi / 4,0)$.

Осталось показать, что точки \pm 1 лежат на внешних линиях Стокса графа $\Gamma(\lambda)$ при $\lambda \in \gamma_{1}$. Вспомним, что внешняя линия Стокса пересекает вешественную ось 
(теорема 3.2). Обозначим точку пересечения $x_{0}$. Допустим, что точки \pm 1 лежат на внутренних линиях Стокса. Из этого следует, что $1<x_{0}$. Рассмотрим точку $\lambda_{0}$, удовлетворяюшую равенству

$$
\frac{1}{\sqrt{\lambda_{0}}}=\frac{x_{0}}{\sqrt{\lambda}}
$$

Эта точка имеет тот же аргумент, что и $\lambda$, но меньший модуль, следовательно, тоже лежит в П, что противоречит единственности.

Доказательство регулярности оставшихся точек $\lambda$ рассматриваемой области непосредственно следует из того, что для них точки \pm 1 не лежат на линиях Стокса, а граф $\Gamma(\lambda)$ состоит из двух компонент. Теорема доказана.

СлеДСТВИЕ 3.1. В случае $\arg \lambda \in(-\pi / 4,0)$ точки \pm 1 не могут лежать $в$ области, ограниченной внутренними линиями Стокса обоих комплексов.

ДокАЗАтЕЛЬСтво. Пусть найдется точка $\lambda^{*}, \arg \lambda^{*} \in(-\pi / 4,0)$, такая, что точки \pm 1 находятся в указанной области. Так как внешняя линия Стокса пересекает $\mathbb{R}^{+}$(теорема 3.2 ), скажем, в точке $x_{2}>1$, то одна из внутренних линий Стокса графа $\Gamma\left(\lambda^{*}\right)$ пересечет $\mathbb{R}^{+}$в точке $x_{1} \in\left(1, x_{2}\right)$.

Если $\psi^{*}=\arg \lambda^{*}$, то обе точки $\left(\frac{1}{x_{2}}\right)^{2} \lambda^{*}$ и $\left(\frac{1}{x_{1}}\right)^{2} \lambda^{*}$ принадлежат П, имеют аргумент $\psi^{*}$ и соответствуюшие им графы Стокса содержат точки \pm 1 . Это противоречит теореме 3.5. Следствие доказано.

TEOPEMA 3.6. Множество сингулярных точек в П состоит из отрезка $\gamma_{0}=\left[0, r_{0} e^{-i \pi / 4}\right]$ и кривой $\gamma_{1}$, определенной в (3.5). В точке $r_{0} e^{-i \pi / 4} \kappa р и$ вые $\gamma_{0}$ и $\gamma_{1}$ пересекаются.

ДоКАЗАТЕЛЬСТво. Первое утверждение является следствием теорем 3.3-3.5. Докажем, что точка $r_{0} e^{-i \pi / 4}$ лежит на $\gamma_{1}$. Согласно определению число $r_{0}$ определяется равенством $r_{0}=|\lambda|$, где число $\lambda, \arg \lambda=-\pi / 4$, удовлетворяет уравнению (3.4). Но тогда из (3.6) следует, что $r_{0} e^{-i \pi / 4} \in \gamma_{1}$. Поэтому $\gamma_{0}$ и $\gamma_{1}$ пересекаются в этой точке. Теорема доказана.

\section{§4. Предельный спектральный граф оператора $L(\varepsilon)$}

Изучим поведение собственных значений оператора $L(\varepsilon)$ при $\varepsilon \rightarrow 0$. В этом параграфе мы докажем, что собственные значения оператора $L(\varepsilon)$ локализуются при $\varepsilon \rightarrow 0$ вблизи отрезка $\gamma_{0}$, кривой $\gamma_{1}$ и кривой $\gamma_{\infty}$, выходящей из точки пересечения $\gamma_{0}$ и $\gamma_{1}$ и уходящей в $-i \infty$. Кривые $\gamma_{0}$ и $\gamma_{1}$ были определены в $\S 3$, а уравнение кривой $\gamma_{\infty}$ укажем ниже.

Сделаем замену $u=1 / \sqrt{\varepsilon}$ и обозначим через $L_{u}$ оператор, соответствуюший получившейся спектральной задаче:

$$
\begin{gathered}
W^{\prime \prime}(z)=u^{2} i\left(z^{2}-\lambda\right) W \\
W(-1)=W(1)=0 .
\end{gathered}
$$

Далее полагаем

$$
\begin{aligned}
& q(\zeta, \lambda)= i\left(\zeta^{2}-\lambda\right), \quad \Psi(z, \lambda)=\int_{\sqrt{\lambda}}^{z} \sqrt{q(\zeta, \lambda)} d \zeta \\
& \widetilde{w}_{1}(z, \lambda, u)=q^{-1 / 4} e^{+u \Psi(z, \lambda)} \\
& \widetilde{w}_{2}(z, \lambda, u)=q^{-1 / 4} e^{-u \Psi(z, \lambda)}
\end{aligned}
$$


Функции $\widetilde{w}_{1}, \widetilde{w}_{2}$ известны как ВКБ-приближения решений уравнения (4.1). Следующие две леммы доказаны в $[5, \S 3$, п. 5$]$.

ЛЕмма 4.1. Пусть $\Omega$ - связный компакт в $\lambda$-плоскости, все точки которого допустимы (в нашем случае в силу леммы 3.2 компакт $\Omega$ не содержит точек $\lambda: \arg \lambda=-\pi / 4)$. Пусть $D(\lambda)$ - канонические области функиии $q(z, \lambda)$, непрерывно зависящие от $\lambda \in \Omega$. Тогда существует фундаментальная система решений $w_{1}, w_{2}$ уравнения (4.1), для которой при $z \in D=\cap_{\lambda \in K} D(\lambda)$ справедливь асимптотики

$$
w_{1,2}(z, \lambda, u)=\widetilde{w}_{1,2}(z, \lambda, u)\left(1+O\left(\frac{1}{u}\right)\right) .
$$

Указанные асимптотики можно дифференцировать по $z, u, \lambda$ любое число pas.

Если $\lambda_{0}$ - недопустимая точка, то может существовать семейство канонических областей $D(\lambda)$, непрерывно зависящее от $\lambda$ при $\lambda \notin I_{3}$, которое при $\lambda \rightarrow \lambda_{0}$ имеет пределом неодносвязную область, следовательно, не каноническую. Но могут также существовать области $D(\lambda)$, которые остаются непрерывными при $\lambda$, принадлежащих всему множеству $I_{3}$ или его части. В такой ситуации лемма 4.1 допускает обобщение.

ЛЕмма 4.2. Пусть $\Omega$ - связный компакт в $\lambda$-плоскости, все точки которого либо допустимь, либо принадлежат типу $I_{3}$ (для рассматриваемого случая функиии $q=x^{2}$ область $\Omega$ не содержит 0 ). Пусть канонические области $D(\lambda)$ таковы, что при $\lambda \in \Omega$ они непрерывны. Тогда справедливо утверждение леммы 4.1 .

Основной смысл следующей леммы состоит в том, что известные асимптотические формулы мы получаем равномерно как по большому параметру $u$, так и по спектральному параметру $\lambda \rightarrow \infty$.

ЛЕмма 4.3. Фиксируем произвольное число $u_{0}>0$ и произвольный компакт $K$ в z-плоскости. Тогда найдется число $R_{0}>0$ такое, что уравнение (4.1) имеет пару независимых решений

$$
\begin{gathered}
U(z)=c q^{-1 / 4} e^{u \Psi(z, \lambda)}\left(1+\frac{\varepsilon_{+}(z, u, \lambda)}{u \lambda}\right), \\
V(z)=c q^{-1 / 4} e^{-u \Psi(z, \lambda)}\left(1+\frac{\varepsilon_{-}(z, u, \lambda)}{u \lambda}\right),
\end{gathered}
$$

где функиия $\Psi(z, \lambda)$ определена равенством $(4.2)$, а функиии $\varepsilon_{ \pm}(z, u, \lambda)$ равномерно ограничены при $z \in K, u>u_{0}, \lambda \in \Pi,|\lambda|>R_{0}$.

ДокАЗАТЕЛЬСтво. В уравнении (4.1) сделаем следующую замену:

$$
y(\eta)=W(\sqrt{\lambda} \eta), \quad \mu=\lambda u e^{\frac{\pi}{4} i},
$$

после которой получим

$$
y^{\prime \prime}=\mu^{2}\left(\eta^{2}-1\right) y \text {. }
$$


Теперь проведем замену Лиувилля

$$
w(\xi)=\sqrt{\xi^{\prime}(z)} y(\eta), \quad \xi(\eta)=\int_{1}^{\eta} \sqrt{\zeta^{2}-1} d \zeta,
$$

которая корректно определена в любой области однолистности функции $\xi(\eta)$. Здесь фиксируем некоторую ветвь $\xi(\eta)$, например, условием $\xi(0)=i \pi / 4$.

Рассмотрим линии Стокса, задаваемые уравнением

$$
\operatorname{Re}\left(e^{-\frac{\pi}{4} i} \xi(\eta)\right)=0 .
$$

Так как $\xi(0)=i \pi / 4$, то $\eta=0$ не лежит на линиях Стокса. Следовательно, найдется $\delta$-окрестность нуля, в которой $\xi(\eta)$ однолистна. Пусть компакт $K$ лежит в круге радиуса $R_{1}$. Из условия $z=\sqrt{\lambda} \eta \in K$ получаем, что при $|\lambda|>R_{1}^{2} / \delta^{2}=R_{0}$ переменная $\eta$ лежит в $\delta$-окрестности нуля. Поэтому при $|\lambda|>R_{0}$ замена Лиувилля корректна. Проведя эту замену, получим уравнение

$$
\begin{gathered}
\frac{d^{2}}{d \xi^{2}} w(\xi)=\left(\mu^{2}+\phi(\xi)\right) w(\xi), \\
\phi(\xi)=\dot{\eta}^{\frac{1}{2}} \frac{d^{2}}{d \xi^{2}}\left(\dot{\eta}^{-\frac{1}{2}}\right)=\frac{1}{2\left(\eta^{2}-1\right)^{2}}-\frac{5 \eta^{2}}{4\left(\eta^{2}-1\right)^{3}}, \quad \eta=\eta(\xi) .
\end{gathered}
$$

Известно [5], что это уравнение имеет пару независимых решений $w_{ \pm}$, для которых справедливы асимптотические оценки

$$
w_{ \pm}(\xi)=e^{ \pm \mu \xi}\left(1+h_{ \pm}(\mu, \xi)\right), \quad\left|h_{ \pm}(\mu, \xi)\right|<\exp \left(\frac{\Phi_{ \pm}(\xi)}{|\mu|}\right)-1
$$

где

$$
\Phi_{ \pm}(\xi)=\int_{\gamma_{ \pm}(\xi)}|\psi(s)||d s|
$$

a $\gamma_{ \pm}(\xi)$ - пути, соединяющие некоторые точки $\xi_{0}^{ \pm}$с $\xi$, причем эти пути выбираются так, что выполняется неравенство

$$
\operatorname{Re}( \pm \mu[\eta-\xi]) \leqslant 0 \quad \text { при всех } \eta \in \gamma_{ \pm}(\xi) .
$$

Так как $\arg \mu \rightarrow-\frac{\pi}{4}$, если $\lambda \in \Pi$ и $\lambda \rightarrow \infty$, а $\xi$ лежит в малой окрестности точки $\xi(0)=i \pi / 4$, то построение таких путей, не зависяших от $\mu$, возможно. Следовательно, $\left|\Phi_{ \pm}\right|<C$, где $C$ не зависит от $\mu$, а потому

$$
\left|h_{ \pm}(\mu, \xi)\right|<\frac{2 C}{u|\lambda|} .
$$

Возвращаясь к исходному уравнению, получим требуемые оценки на функции $\varepsilon_{ \pm}$. Лемма доказана.

В теореме 3.6 было определено множество сингулярных точек, состоящее из отрезка $\gamma_{0}$ и кривой $\gamma_{1}$. Теперь рассмотрим кривую

$$
\gamma_{\infty}=\left\{\lambda \in \Pi \mid \operatorname{Re}\left(e^{\frac{\pi}{4} i} \int_{-1}^{1} \sqrt{\zeta^{2}-\lambda} d \zeta\right)=0 \& \arg \lambda \in\left(-\frac{\pi}{2},-\frac{\pi}{4}\right]\right\} .
$$



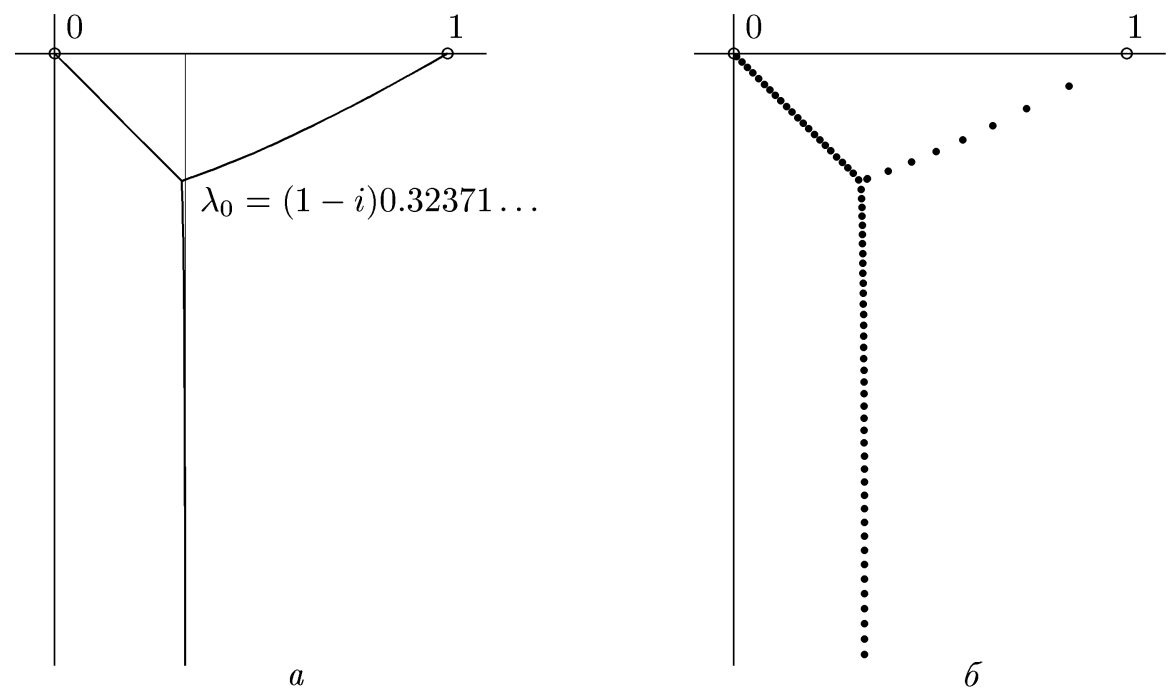

Рис. 6. $a$-предельные спектральные кривые $\gamma=\gamma_{0} \cup$ $\gamma_{1} \cup \gamma_{\infty}, 6$ - спектр оператора $L(\varepsilon)$ при $\varepsilon=10^{-4}$

Из определения кривых $\gamma_{0}, \gamma_{1}$ и $\gamma_{\infty}$ следует, что точка $r_{0} e^{-i \pi / 4}$ является их общей точкой пересечения. Рассмотрим множество (см. рис. 6)

$$
\gamma=\gamma_{0} \cup \gamma_{1} \cup \gamma_{\infty}
$$

которое назовем предельным спектральным графом оператора $L(\varepsilon)$. Ниже будет показано, что $\gamma$ является множеством, вблизи которого концентрируются собственные значения оператора $L(\varepsilon)$. Но сначала покажем, что $\gamma_{\infty}$ асимптотически приближается к прямой $\operatorname{Re} \lambda=1 / 3$.

ЛЕмма 4.4. При $\lambda \in \gamma_{\infty}$ справедливо асимптотическое равенство

$$
\operatorname{Re}\left(e^{\frac{\pi}{4} i} \int_{-1}^{1} \sqrt{\zeta^{2}-\lambda} d \zeta\right)=\frac{1}{|\lambda|^{1 / 2}}\left(\frac{1}{3}-\operatorname{Re} \lambda\right)+O\left(\frac{1}{|\lambda|^{3 / 2}}\right), \quad \lambda \rightarrow \infty
$$

В частности, точки $\lambda \in \gamma_{\infty}$ асимптотически приближаются $\kappa$ прямой $\operatorname{Re} \lambda=1 / 3$ со скоростью $O\left(|\lambda|^{-1}\right)$. 
ДокаЗАТЕЛЬСТво. Пусть $\psi=\arg \lambda, r=|\lambda|$. Имеем

$$
\begin{aligned}
& \operatorname{Re}\left(e^{\frac{\pi}{4} i} \int_{-1}^{1} \sqrt{\zeta^{2}-\lambda} d \zeta\right)=2 \operatorname{Re}\left(e^{\frac{\pi}{4} i} \int_{0}^{1} \sqrt{\zeta^{2}-\lambda} d \zeta\right) \\
& \quad=2 \operatorname{Re}\left(e^{\frac{3 \pi}{4} i}\left(\sqrt{\lambda}-\frac{1}{6 \sqrt{\lambda}}+O\left(\frac{1}{\lambda^{3 / 2}}\right)\right)\right) \\
& \quad=2 r^{1 / 2}\left(\cos \left(\frac{3 \pi}{4}+\frac{\psi}{2}\right)-\frac{1}{6 r^{1 / 2}} \cos \left(\frac{3 \pi}{4}-\frac{\psi}{2}\right)\right)+O\left(\frac{1}{r^{3 / 2}}\right) \\
& \quad=2\left(-r^{1 / 2} \sin \left(\frac{\pi}{4}+\frac{\psi}{2}\right)+\frac{1}{6 r^{1 / 2}} \cos \left(\frac{\pi}{4}+\frac{\psi}{2}\right)\right)+O\left(\frac{1}{r^{3 / 2}}\right) \\
& \quad=r^{-1 / 2} \cos \left(\frac{\pi}{4}+\frac{\psi}{2}\right)\left(\frac{1}{3}-2 r \operatorname{tg}\left(\frac{\pi}{4}+\frac{\psi}{2}\right)\right)+O\left(\frac{1}{r^{3 / 2}}\right) \\
& \quad=r^{-1 / 2} \cos \left(\frac{\pi}{4}+\frac{\psi}{2}\right)\left(\frac{1}{3}-\frac{\operatorname{Re} \lambda}{\cos ^{2}\left(\frac{\pi}{4}+\frac{\psi}{2}\right)}\right)+O\left(\frac{1}{r^{3 / 2}}\right)
\end{aligned}
$$

Далее, имеем $\cos \left(\frac{\pi}{4}+\frac{\psi}{2}\right)=1+O\left(r^{-1}\right)$ при $\lambda=r e^{i \psi} \rightarrow \infty$ внутри полуполосы $\Pi$ (в этом случае $\psi \rightarrow-\pi / 2$ ). Из этого соотношения следует утверждение леммы.

ЗАмЕчАниЕ 4.1. Утверждение леммы сохраняет силу не только для $q(x)=x^{2}$, а для произвольной целой функции $q$. Только число $1 / 3$ нужно заменить на число $\int_{-1}^{1} q(x) d x$.

TEOPEMA 4.1. Пусть $\gamma(\delta)$ - -окрестность множества $\gamma$. Тогда для любого $\delta>0$ найдется число $\varepsilon_{0}>0$ такое, что все собственные значения оператора $L(\varepsilon)$ при $\varepsilon<\varepsilon_{0}$ лежат внутри $\gamma(\delta)$.

ДокаЗАТЕЛЬСтво. Множество $\Pi \backslash \gamma(\delta)$ состоит из трех связных замкнутых попарно не пересекающихся компонент. Обозначим их через $\Lambda_{l}, \Lambda_{r}, \Lambda_{+}$(левая, правая и верхняя соответственно). В наших обозначениях $\varepsilon=u^{-2}$, поэтому нужно доказать, что найдется число $u_{0}$ такое, что при $u>u_{0}$ характеристический определитель

$$
\Delta(u, \lambda)=\left|\begin{array}{ll}
U(-1) & U(1) \\
V(-1) & V(1)
\end{array}\right|
$$

отличен от нуля, где $U$ и $V$ - пара линейно независимых решений уравнения (4.1). Рассмотрим отдельно три случая, когда $\lambda$ принадлежит одной из компонент $\Lambda_{l}, \Lambda_{r}$ или $\Lambda_{+}$.

1. Пусть $\lambda \in \Lambda_{l}$. Предположим, что утверждение теоремы неверно. Тогда найдутся последовательности $u_{n} \rightarrow+\infty$ и $\lambda_{n} \in \Lambda_{l}$ такие, что

$$
\Delta\left(u_{n}, \lambda_{n}\right)=0 .
$$

Сначала покажем, что последовательность $\lambda_{n}$ ограничена. При $\lambda \in \Lambda_{l},|\lambda|>R_{0}$, можно воспользоваться леммой 4.3. Тогда получаем, что уравнение $\Delta(u, \lambda)=0$ эквивалентно уравнению

$$
e^{2 u(\Psi(1, \lambda)-\Psi(-1, \lambda))}=1+\frac{\varepsilon(u, \lambda)}{u \lambda},
$$

7

Серия математическая, №4 
где $|\varepsilon(u, \lambda)|<$ const при $u>u_{0}$ и $|\lambda|>R_{0}$.

Заметим, что функция $Q=\sqrt{i\left(z^{2}-\lambda\right)}$ допускает выделение однозначных голоморфных ветвей в плоскости $\mathbb{C}$ с разрезами по внешним линиям Стокса. Согласно теореме 3.1 в случае $\arg \lambda \in(-\pi / 2,-\pi / 4)$ (тем более, при $\left.\lambda \in \Lambda_{l}\right)$ внешние линии Стокса не пересекают вешественную ось. Но тогда два интеграла от $\sqrt{\lambda}$ до \pm 1 можно заменить одним по отрезку $[-1,1]$, т. е. справедливо равенство

$$
T(\lambda)=\Psi(1, \lambda)-\Psi(-1, \lambda)=e^{\frac{\pi}{4} i} \int_{-1}^{1} \sqrt{\zeta^{2}-1} d \zeta
$$

Далее, в силу леммы 4.4

$$
\left|\operatorname{Re}\left(e^{\frac{\pi}{4} i} \int_{-1}^{1} \sqrt{\zeta^{2}-1} d \zeta\right)\right|>\frac{C}{|\lambda|^{1 / 2}}, \quad \text { если } \lambda \in \Lambda_{l} .
$$

Сравнение этого неравенства с (4.4) приводит к противоречию. Следовательно, последовательность $\lambda_{n}$ ограничена и лежит в некотором компакте $K \subset \Lambda_{l}$. Воспользовавшись леммой 4.1, как и ранее, получим, что уравнение $\Delta(u, \lambda)=0$ эквивалентно уравнению

$$
e^{2 u(\Psi(1, \lambda)-\Psi(-1, \lambda))}=1+O(1 / u) .
$$

Но в силу равенства (4.5) имеем

$$
|\operatorname{Re} T(\lambda)|>C=C(\delta)
$$

при $\lambda \in \Lambda_{l}$, что противоречит (4.6), если $u>u_{0}$, где $u_{0}$ - достаточно большое число. Поэтому в области $\Lambda_{l}$ отсутствуют собственные значения оператора $L(\varepsilon)$, если $u>u_{0}$.

2. Точно так же, как в предыдушем случае, можно показать, что в области $\Lambda_{r}$ при $|\lambda|>R_{0}$ собственных значений нет. Рассмотрим компакт

$$
\Lambda_{r}^{\prime}=\left\{\lambda \in \Lambda_{r}|| \lambda \mid \leqslant R_{0}\right\} .
$$

Пусть $\lambda \in \Lambda_{r}^{\prime}$ и $\arg \lambda<-\pi / 4$. Как и ранее, в этом случае имеем

$$
|\operatorname{Re} T(\lambda)|>c(\lambda)>0 .
$$

Если $\lambda \in \Lambda_{r}^{\prime}$ и $\arg \lambda>-\pi / 4$, то представление (4.5) уже неверно (оно будет верно, если вместо интеграла по отрезку $[-1,1]$ рассматривать интеграл по контуру $\gamma_{\lambda}$, огибающему точки $\sqrt{\lambda}$ и $-\sqrt{\lambda}$ сверху и снизу соответственно). Однако в силу следствия 3.1 точки \pm 1 лежат в одной канонической области и отделены внутренними линиями Стокса, а потому функции $\operatorname{Re} \Psi(1, \lambda)$ и $\operatorname{Re} \Psi(-1, \lambda)$ имеют разные знаки. Следовательно, в рассматриваемом случае также справедливо неравенство (4.7). Наконец, если $\lambda \in \Lambda_{r}^{\prime}$ и $\arg \lambda=-\pi / 4$, то точки \pm 1 лежат в одной канонической области и отделены линией Стокса. Поэтому в этом случае функции $\operatorname{Re} \Psi(1, \lambda)$ и $\operatorname{Re} \Psi(-1, \lambda)$ также имеют разные знаки и выполнена оценка (4.7). Кроме того, точки $\lambda \in \Lambda_{r}^{\prime}$ являются регулярными. Поэтому в силу леммы 4.2 функция $T(\lambda)$ 
является однозначной при $\lambda \in \Lambda_{r}$ (здесь нужно иметь в виду, что $T(\lambda)$ определяется при $\arg \lambda<-\pi / 4$ и $\arg \lambda>-\pi / 4$ различньм образом, но предельные значения снизу и сверху на луче $\arg \lambda=-\pi / 4$ совпадают).

Таким образом, неравенство (4.7) выполнено для всех $\lambda$ на компакте $\Lambda_{r}^{\prime}$. Но тогда $|c(\lambda)| \geqslant c_{0}>0$ при $\lambda \in \Lambda_{r}^{\prime}$, а потому $\Lambda_{r}^{\prime}$ не содержит собственных значений при больших значениях $u$.

3. Все точки компакта $\Lambda^{+}$являются регулярными и допустимыми. Поэтому можно найти контур, соединяюший точки \pm 1 в $z$-плоскости, который будет принадлежать пересечению канонических областей $D(\lambda)$ графа Стокса $\Gamma(\lambda)$ при всех $\lambda \in \Lambda^{+}$. Точки \pm 1 в областях $D(\lambda)$ разделяются линией Стокса, поэтому функции $\operatorname{Re} \Psi(1, \lambda)$ и $\operatorname{Re} \Psi(-1, \lambda)$ имеют разные знаки. Следовательно, равенство (4.5) не выполняется при больших значениях $u$. Теорема доказана.

Из теоремы 4.1 и леммы 4.4 легко получаем следующий результат, уточняющий предложение 2.2 .

СлеДСТВИЕ 4.1. Спектр оператора $L(\varepsilon)$ равномерно при $\varepsilon>0 u|\lambda|>r_{0}$ приближается $к$ прямой $\operatorname{Re} \lambda=1 / 3$. Более того, для точек $\lambda$, принадлежащих спектру $L(\varepsilon)$, выполнена оценка

$$
\left|\frac{1}{3}-\operatorname{Re} \lambda\right| \leqslant \frac{C \varepsilon}{|\lambda|^{1 / 2}}, \quad|\lambda|>r_{0},
$$

где постоянная $C$ не зависит от $\lambda u \varepsilon$.

\section{$\S 5$. Поведение спектра при $\varepsilon \rightarrow 0$}

Далее нам потребуются утверждения о матрицах перехода, доказательства которых можно найти в монографии [5].

Пусть даны две пары $\left(U_{j}, V_{j}\right),\left(U_{k}, V_{k}\right)$ линейно независимых решений уравнения (4.1). Здесь индексы $j$ и $k$ фиксированы. Произвольное решение $W$ этого уравнения можно представить линейной комбинацией этих пар решений:

$$
W=\alpha_{j} U_{j}+\beta_{j} V_{j}=\alpha_{k} U_{k}+\beta_{k} V_{k} .
$$

ОПРЕДЕЛЕНИЕ 5.1. Матрица $\Omega_{j k}=\Omega_{j k}(u, \lambda)$ такая, что

$$
\left(\begin{array}{c}
\alpha_{k} \\
\beta_{k}
\end{array}\right)=\Omega_{j k}\left(\begin{array}{c}
\alpha_{j} \\
\beta_{j}
\end{array}\right)
$$

для всех решений $W$ уравнения (4.1), называется матрицей перехода от пары $\left(U_{j}, V_{j}\right) \kappa$ nаре $\left(U_{k}, V_{k}\right)$. В частности, при всех $z \in \mathbb{C}$ справедливо равенство

$$
\left(\begin{array}{c}
U_{j} \\
V_{j}
\end{array}\right)=\Omega_{j k}^{T}\left(\begin{array}{c}
U_{k} \\
V_{k}
\end{array}\right)
$$

где $\Omega_{j k}^{T}$ - транспонированная матрица перехода.

Как и ранее, положим $q(z, \lambda)=i\left(z^{2}-\lambda\right)$. Рассмотрим каноническую тройку $\left(D, l, z_{0}\right)$ и каноническую ветвь $S$ (в смысле определений $\left.3.5,3.6\right)$. Удалим из $S(D)$ левые (правые) $\varepsilon$-окрестности разрезов и $\varepsilon$-окрестности образов точек поворота. Прообраз обозначим через $D_{\varepsilon}^{+}\left(D_{\varepsilon}^{-}\right)$. 
ОПРЕДЕЛЕНИЕ 5.2. Бесконечньй путь $\gamma^{+}(z) \subset D\left(\gamma^{-}(z) \subset D\right)$ назьвается noложительным (отрицательньм) каноническим путем для тройки $\left(D, l, z_{0}\right)$, если один из его концов совпадает с $z$ и для канонической ветви $S$ имеет место соотношение $\operatorname{Re} S\left(\zeta, z_{0}\right) \rightarrow+\infty(-\infty)$ при $\zeta \in \gamma^{+}(z)\left(\zeta \in \gamma^{-}(z)\right), z \rightarrow \infty$.

ЛЕмма 5.1. Пусть $\left(D, l, z_{0}\right)$ - каноническая тройка, $S$ - каноническая ветвъ. Тогда при и 1 в области $D$ существует пара независимых решений $(U, V)$, именуемая канонической парой, соответствующей данной канонической тройке, асимптотически представляемых в $D$ разложениями при $u \rightarrow+\infty$ :

$$
\begin{aligned}
& U(z, u) \sim c q^{-1 / 4}(z) e^{+u S\left(z, z_{0}\right)} \exp \left(\sum_{k=1}^{\infty}(+u)^{-k} \int_{\gamma^{+}(z)} \alpha_{k}(t) d t\right) \\
& V(z, u) \sim c q^{-1 / 4}(z) e^{-u S\left(z, z_{0}\right)} \exp \left(\sum_{k=1}^{\infty}(-u)^{-k} \int_{\gamma^{-}(z)} \alpha_{k}(t) d t\right)
\end{aligned}
$$

əде

$$
\alpha_{1}=\frac{1}{8} \frac{q^{\prime \prime}}{q^{3 / 2}}-\frac{5}{32} \frac{\left(q^{\prime}\right)^{2}}{q^{5 / 2}}, \quad \alpha_{k+1}=-\frac{1}{2 \sqrt{q}}\left(\alpha_{k}^{\prime}+\sum_{j=0}^{k} \alpha_{j} \alpha_{k-j}\right)
$$

и для удобства записи используются обозначения

$$
\alpha_{-1}=\sqrt{q}, \quad \alpha_{0}=-\frac{q^{\prime}}{4 q}
$$

Здесь $\gamma^{ \pm}(z)$ - произвольные положительный и отрицательный канонические пути в $D$, а с - нормировочный коэффициент такой, что

$$
|c|=1, \quad \lim _{z \rightarrow z_{0}, z \in l} \arg \left(c q^{-1 / 4}(z)\right)=0 .
$$

ОПРЕДЕЛЕНИЕ 5.3. Пусть заданы две канонические тройки $\left(D_{j}, l_{j}, z_{j}\right)$ и $\left(D_{k}\right.$, $\left.l_{k}, z_{k}\right)$. Рассмотрим канонические пары решений $\left(U_{j}, V_{j}\right),\left(U_{k}, V_{k}\right)$. Матрицу перехода от $\left(U_{j}, V_{j}\right)$ к $\left(U_{k}, V_{k}\right)$ будем называть матричей перехода от канонической тройки $\left(D_{j}, l_{j}, z_{j}\right) \kappa\left(D_{k}, l_{k}, z_{k}\right)$.

Можно доказать существование четырех типов матрищ перехода между каноническими тройками, из которых путем композиций получаются все другие возможные матрицы перехода. Сформулируем леммы, представляюшие вид матриц в трех специальных ситуациях.

Лемма 5.2. Пусть $\left(D, l, z_{1}\right)$ - первая тройка, $\left(D, l, z_{2}\right)$ - вторая. Меняется лищь направление конечной линии Cтокса l. Тогда

$$
\Omega=e^{i \varphi_{0}}\left(\begin{array}{cc}
0 & e^{-i u a} \\
e^{i u a} & 0
\end{array}\right), \quad a=\left|S\left(z_{1}, z_{2}\right)\right|, \quad e^{i \varphi_{0}}=c_{2} / c_{1} .
$$


Лемма 5.3. Пусть $\left(D, l_{1}, z_{1}\right)$ - первая тройка, $\left(D, l_{2}, z_{2}\right)$ - вторая, причем лучи $S\left(l_{1}\right)$ и $S\left(l_{2}\right)$ направлены в одну сторону. Пусть $l_{2}$ находится слева om $l_{1}, a=S\left(z_{1}, z_{2}\right)$, Re $a>0$. Tогдa

$$
\Omega=e^{i \varphi_{0}}\left(\begin{array}{cc}
e^{-u a} & 0 \\
0 & e^{u a}
\end{array}\right), \quad e^{i \varphi_{0}}=c_{2} / c_{1} .
$$

ЛЕмма 5.4. Пусть $z_{0}$ - простая точка поворота (нуль первого порядка функции q), $l_{1}, l_{2}, l_{3}$ - линии Стокса с началом $z_{0}, l_{j+1}$ лежит слева от $l_{j}$. Пусть канонические тройки $\left(D_{j}, l_{j}, z_{0}\right)$ выбраны так, что часть $D_{j}$, лежащая слева от $l_{j}$, совпадает с частью $D_{j+1}$, лежащей справа от $l_{j+1}$. Обозначим матричу перехода от $\left(D_{j}, l_{j}, z_{0}\right) \kappa\left(D_{j+1}, l_{j+1}, z_{0}\right)$ через $\Omega_{j, j+1}$. Тогда

$$
\Omega_{j, j+1}=e^{-\frac{\pi}{6} i}\left(\begin{array}{cc}
0 & \alpha_{j, j+1}^{-1} \\
1 & i \alpha_{j+1, j+2}
\end{array}\right), \quad \alpha_{12} \alpha_{23} \alpha_{31}=1,
$$

и справедливо асимптотическое разложение

$$
\alpha_{j, j+1} \asymp \exp \left(\sum_{k=1}^{\infty}(-u)^{-k} \int_{\gamma_{j, j+1}} \alpha_{k}(t) d t\right) .
$$

Здесь бесконечный контур $\gamma_{j, j+1}$ лежит в $D_{j} \cup D_{j+1}$, начинается в $D_{j+1}$ там, где $\operatorname{Re} S \rightarrow+\infty$, и заканчивается в $D_{j}$ там, где $\operatorname{Re} S \rightarrow-\infty$. Ветвь $\sqrt{q}$ выбрана так же, как для фундаментальной системы $\left(U_{j}, V_{j}\right)$.
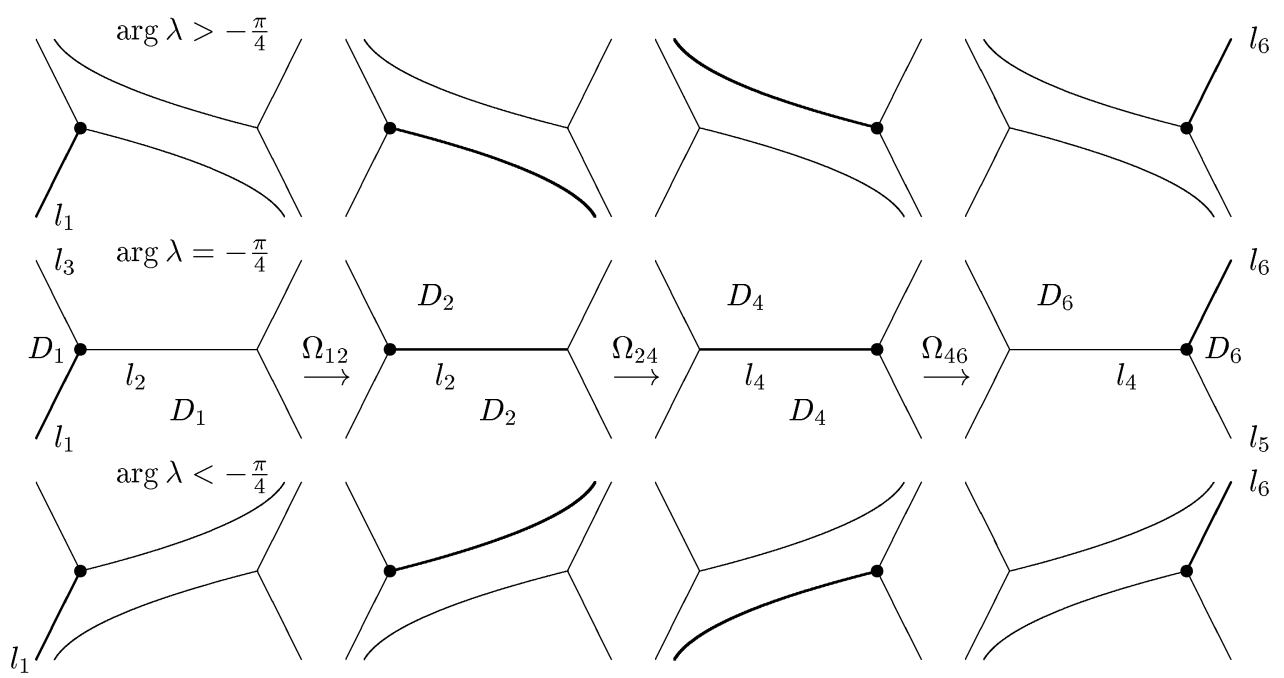

Рис. 7. Матрицы перехода и канонические области для решений в окрестности $\gamma_{0}$ 
Перейдем к изучению поведения спектра вблизи кривых $\gamma_{0}, \gamma_{1}$ и $\gamma_{\infty}$. Наиболее подробно изучим локализацию спектра вблизи $\gamma_{0}$; доказательства для $\gamma_{1}, \gamma_{\infty}$ оказываются проше.

При движении вдоль пути, пересекающего отрезок $\gamma_{0}$, граф претерпевает изменения, показанные на рис. 7 (для определенности считаем, что аргумент при движении строго убывает). Верхний ряд соответствует тем точкам кривой, для котоpых $\arg \lambda>-\pi / 4 ;$ средний ряд - для $\arg \lambda=-\pi / 4 ;$ нижний - для $\arg \lambda<-\pi / 4$. Обратим внимание, что все канонические области, обозначенные на рис. 7 , непрерывно зависят от $\lambda$, а потому выполняются условия леммы 4.2. Следовательно, равномерными по $\lambda$ являются асимптотики для матрищ перехода в любом компакте в П, не содержашем 0 .

Обозначим линии Стокса и канонические тройки так, как это сделано на рис. 7. Индексы канонических решений положим равными индексам канонических областей.

Лемма 5.5. Матрица перехода $\Omega_{16}$ от канонической тройки $\left(D_{1}, l_{1},-\sqrt{\lambda}\right)$ $\kappa$ тройке $\left(D_{6}, l_{6}, \sqrt{\lambda}\right)$ выражсается следующей формулой:

$$
\begin{gathered}
\Omega_{16}^{T}=\left(\begin{array}{cc}
\omega_{11} & \omega_{12} \\
\omega_{21} & \omega_{22}
\end{array}\right)=C\left(\begin{array}{cc}
-i \alpha_{45} \alpha_{64} e^{-i u a} & \alpha_{64} e^{-i u a} \\
\alpha_{23} \alpha_{45} \alpha_{64} e^{-i u a}+\frac{1}{\alpha_{12}} e^{i u a} & i \alpha_{23} \alpha_{64} e^{-i u a}
\end{array}\right), \\
a=|\lambda| \frac{\pi}{2}
\end{gathered}
$$

Элементы $\omega_{i j}$ продолжаются аналитически в окрестность отрезка $\gamma_{0} c$ выколотой окрестностью нуля, а их асимптотики равномерны в указанной области.

ДокАЗАТЕЛьство. Согласно схеме, изображенной на рис. 7 , имеем

$$
\Omega_{16}^{T}=\Omega_{12}^{T} \Omega_{24}^{T} \Omega_{46}^{T}=\Omega_{12}^{T} \Omega_{24}^{T}\left(\Omega_{64}^{T}\right)^{-1} .
$$

Вид матрищ в правой части указан в леммах 5.2 и 5.4. После последовательного умножения этих матриц получаем (5.2). Аналитичность элементов матриц следует из аналитичности канонических решений по параметру $\lambda$, а равномерность следует из лемм 4.1 и 4.2. Имеем также

$$
a=\left|e^{\frac{\pi}{4} i} \lambda \int_{-1}^{1} \sqrt{\zeta^{2}-1} d \zeta\right|=|\lambda| \frac{\pi}{2} .
$$

Лемма доказана.

Обозначим через $\Upsilon$ малую окрестность отрезка $\gamma_{0}$ с выколотыми окрестностями нуля и точки-узла $\lambda_{0}=r_{0} e^{-i \pi / 4}$. Характеристический определитель $\Delta(u, \lambda)$ в области $\Upsilon$ имеет представление

$$
\begin{aligned}
& \Delta(u, \lambda)=\left|\begin{array}{ll}
U_{1}(-1) & U_{1}(1) \\
V_{1}(-1) & V_{1}(1)
\end{array}\right|=\left|\begin{array}{ll}
U_{1}(-1) & \omega_{11} U_{6}(1)+\omega_{12} V_{6}(1) \\
V_{1}(-1) & \omega_{21} U_{6}(1)+\omega_{22} V_{6}(1)
\end{array}\right| \\
& =\omega_{21} U_{1}(-1) U_{6}(1)+\omega_{22} U_{1}(-1) V_{6}(1)-\omega_{11} V_{1}(-1) U_{6}(1)-\omega_{12} V_{1}(-1) V_{6}(1)
\end{aligned}
$$

Рассмотрим канонические ветви $\Psi_{6}(1,-\lambda)$ и $\Psi_{1}(-1, \lambda)$. 
ЛЕмма 5.6. Область $\Upsilon$, содержащую $\gamma_{0}$ с выколотыми точками 0 и $\lambda_{0}$, можно выбрать столь малой, что для всех $\lambda \in \Upsilon$ выполнены неравенства

$$
\operatorname{Re} \Psi_{1}(-1,-\lambda)>0, \quad \operatorname{Re} \Psi_{6}(1, \lambda)>0 .
$$

ДокАЗАТЕЛЬСТво. Достаточно вспомнить расположение точек \pm 1 относительно графа Стокса при $\lambda \in \Upsilon$ и воспользоваться тем, что рассматриваемые функции голоморфны при $\lambda \in \Upsilon$.

Лемма 5.7. Пусть $\gamma_{0}^{\prime}$ - замкнутая часть отрезка $\gamma_{0}$, не содержащая его концов. Тогда найдутся малая окрестность $\Upsilon$ кривой $\gamma_{0}^{\prime}$ и числа $u_{0}>0$, $C>0$ mакие, что при всех $u>u_{0}$ область $\Upsilon$ будет содержать точки спектра. Более того, если рассмотреть точки $\tilde{\lambda}_{k}=e^{-i \pi / 4}(2 k+1) / u, k \in \mathbb{N}$, то в каждой окрестности $\mathscr{U}_{k}$ этих точек радиуса $C / u^{2}$, лежащей в $\Upsilon$, найдется и притом единственная точка спектра. При всех $и>u_{0}$ других точек спектра $\lambda \in \Upsilon$, лежащих вне окрестности $\mathscr{U}_{k}$, нет.

ДокаЗАТЕЛЬСтво. Рассмотрим область $\Upsilon$ такую, что $\gamma_{0}^{\prime} \subset \Upsilon$, причем при $\lambda \in \Upsilon$ выполнены неравенства (5.3). Из леммы 5.6 следует, что при $u \rightarrow+\infty$ равномерно в $\Upsilon$ справедливо асимптотическое равенство

$$
\Delta(u, \lambda)=\omega_{21} q^{-1 / 4}(1) q^{-1 / 4}(-1) e^{u\left(\Psi_{6}(1, \sqrt{\lambda})+\Psi_{1}(-1, \sqrt{\lambda})\right)}\left(1+o\left(e^{-u \delta}\right)\right), \quad \delta>0 .
$$

Поэтому равенство $\Delta(u, \lambda)=0$ эквивалентно уравнению, справедливому в $\Upsilon$ равномерно при $u \gg 1$ :

$$
\omega_{21}=0 \text {. }
$$

Согласно лемме 5.5 имеем

$$
\omega_{21}=\frac{\alpha_{23}}{\alpha_{56}} e^{-i u a}+\frac{1}{\alpha_{12}} e^{i u a}=0 \Longleftrightarrow e^{2 i u a}=-\frac{\alpha_{31}}{\alpha_{56}}, \quad a=|\lambda| \frac{\pi}{2} .
$$

Обратим внимание на то, что мы дважды воспользовались мультипликативным тождеством для $\alpha_{j k}$ (см. лемму 5.4). Отметим, что формулы, выписанные выше, справедливы лишь на $\gamma_{0}$. При $\lambda \in \gamma_{0}$ можно записать $a=\left(\lambda \pi e^{i \pi / 4}\right) / 2$, т.е. $a$ есть линейная функция на $\gamma_{0}$. Функция $a_{31} / a_{36}$ аналитически продолжается в область $\Upsilon$, причем из (5.1) следует, что $a_{31} / a_{36}=1+O\left(u^{-1}\right)$. Поэтому, аналитически продолжая функцию $\omega_{21}$ в область $\Upsilon$, получаем уравнение

$$
e^{\pi i u e^{i \pi / 4} \lambda}+1=O\left(\frac{1}{u}\right)
$$

на собственные значения, которое решается стандартным методом. А именно, невозмушенное уравнение (без добавочного члена $\left.O\left(u^{-1}\right)\right)$ имеет решения

$$
\tilde{\lambda}_{k}=\frac{2 k+1}{u} e^{-i \pi / 4}
$$


Пусть $\mathscr{U}_{k}$ - окрестность точки $\tilde{\lambda}_{k}$ радиуса $C u^{-2}$, где $C$ - постоянная, которую мы определим позже. В окрестности $\mathscr{U}_{k}$ уравнение (5.4) перепишем в виде

$$
F(\lambda, u):=e^{\pi i u e^{i \pi / 4}\left(\lambda-\tilde{\lambda}_{k}\right)}-1=O\left(\frac{1}{u}\right)
$$

Заметим, что при $\left|\mu-\mu_{k}\right|=C u^{-2}$ в комплексной $\mu$-плоскости справедлива оценка

$$
\left|e^{i \pi\left(\mu-\mu_{k}\right) u}-1\right| \geqslant \frac{\delta C}{u}, \quad u>u_{0},
$$

где постоянная $\delta$ зависит только от $u_{0}$. Следовательно, на границе окрестности $\mathscr{U}_{k}$ радиуса $C u^{-2}$ выполняется оценка

$$
|F(\lambda, u)| \geqslant \frac{\delta C}{u}
$$

Правая часть уравнения (5.5) оценивается величиной $C_{1} u^{-1}$. Выберем постоянную $C$ так, что $\delta C>C_{1}$. Воспользовавшись теоремой Руше, получим, что уравнение (5.5) имеет внутри окрестности $\mathscr{U}_{k}$ столько же нулей, сколько функция $F(\lambda, u)$, т. е. ровно один нуль. Из (5.5) также получаем, что других нулей в области $\Upsilon$, кроме нулей $\lambda_{k}$, близких к $\tilde{\lambda}_{k}$, нет. Лемма доказана.

Для анализа спектра в окрестностях кривых $\gamma_{1}$ и $\gamma_{\infty}$ понадобятся некоторые утверждения о конформности функций, определяюших эти множества. Обозначим

$$
K(\lambda)=\int_{-1}^{1} \sqrt{\zeta^{2}-\lambda} d \zeta, \quad J(\lambda)=\int_{\sqrt{\lambda}}^{1} \sqrt{\zeta^{2}-\lambda} d \zeta
$$

Функцию $K$ считаем определенной в той части П, в которой $\arg \lambda<-\pi / 4$, а функцию $J$ рассматриваем в малой окрестности кривой $\gamma_{1}$.

ЛЕмма 5.8. Функиия $K$ является однолистной в области $D=\{\lambda \in \Pi \mid$ $\left.\arg \lambda<-\frac{\pi}{4}\right\}$.

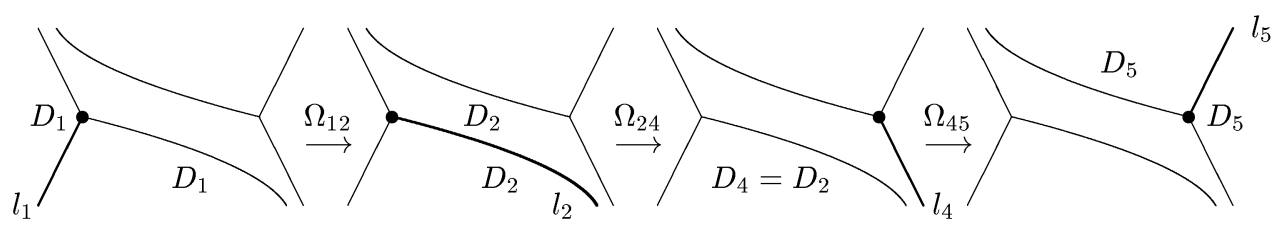

Рис. 8. Матрицы перехода и канонические области для решений в окрестности кривой $\gamma_{1}$ 
ДокАЗАТЕЛЬСТво. Предположим противное: найдутся точки $\lambda_{1} \neq \lambda_{2}$ в $D$ такие, что $K\left(\lambda_{1}\right)=K\left(\lambda_{2}\right)$. Тогда

$$
\int_{-1}^{1}\left(\sqrt{\zeta^{2}-\lambda_{1}}-\sqrt{\zeta^{2}-\lambda_{2}}\right) d \zeta=\int_{-1}^{1}\left(\frac{\lambda_{2}-\lambda_{1}}{\sqrt{\zeta^{2}-\lambda_{1}}+\sqrt{\zeta^{2}-\lambda_{2}}}\right) d \zeta=0 .
$$

Последнее равенство можно сократить на $\lambda_{2}-\lambda_{1}$. При всех $\zeta \in[-1,1], \lambda \in D$ имеем

$$
\sqrt{\zeta^{2}-\lambda} \in \Omega=\left\{z \in \mathbb{C} \mid \arg z \in\left(0, \frac{\pi}{2}\right)\right\} .
$$

Если $\alpha, \beta \in \Omega$, то $\alpha+\beta \in \Omega$, поэтому при всех $\zeta \in[-1,1]$ значения функции

$$
h(\zeta)=\frac{1}{\sqrt{\zeta^{2}-\lambda_{1}}+\sqrt{\zeta^{2}-\lambda_{2}}}
$$

лежат в четвертом квадранте комплексной плоскости, т.е. $\arg h(\zeta) \in(-\pi / 2,0)$. Кроме того, $h(\zeta) \neq 0$, поэтому

$$
\int_{-1}^{1} h(\zeta) d \zeta \neq 0
$$

Полученное противоречие доказывает лемму.

ЛЕмма 5.9. Функция $J$ является однолистной в некоторой малой окрестности кривой $\gamma_{1} \subset \Pi$, но вне малой окрестности точки 1.

ДокАЗАтЕльСтво. Заметим, что $\gamma_{1}$ - аналитическая кривая. Кроме того, непосредственной проверкой легко убедиться в том, что

$$
\frac{\partial \operatorname{Re}\left(e^{\pi i / 4} J(\lambda)\right)}{\partial \operatorname{Re} \lambda} \neq 0
$$

Отсюда получаем утверждение леммы.

Переходим к изучению спектра в окрестности $\gamma_{1}$. Используем те же рассуждения, что и в случае $\gamma_{0}$. Обратимся к схеме, изображенной на рис. 8 , где показаны канонические тройки и используемые матрищы перехода.

Лемма 5.10. Матрица перехода $\Omega_{15}$ от канонической тройки $\left(D_{1}, l_{1},-\sqrt{\lambda}\right)$ $\kappa$ тройке $\left(D_{5}, l_{5}, \sqrt{\lambda}\right)$ выражается формулой

$$
\Omega_{15}^{T}=\left(\begin{array}{ll}
\omega_{11} & \omega_{12} \\
\omega_{21} & \omega_{22}
\end{array}\right)=C\left(\begin{array}{cc}
\frac{e^{u a}}{\alpha_{45}} & e^{u a} i \alpha_{56} \\
i \frac{\alpha_{23}}{\alpha_{45}} e^{u a} & \frac{e^{-u a}}{\alpha_{12}}-\alpha_{23} \alpha_{56} e^{u a}
\end{array}\right), \quad a=\lambda \frac{\pi}{2} i .
$$

Пусть $\Upsilon$ - область, получающаяся из малой окрестности кривой $\gamma_{1}$ удалением мальх окрестностей точек 1 и $r_{0} e^{-i \pi / 4}$. Тогда әлементы $\omega_{i j}$ продолжаются аналитически в область $\Upsilon$, а их асимптотики равномерны в этой области. 
ДокАЗАТЕЛЬСТво. Имеем $\Omega_{15}^{T}=\Omega_{12}^{T} \Omega_{24}^{T} \Omega_{45}^{T}$. Воспользовавшись леммами 5.3, 5.4 и перемножив матрицы, получим представление (5.7).

Лемма 5.11. Пусть $\gamma_{1}^{\prime}$ - замкнутая часть кривой $\gamma_{1}$, не содержащая кон-

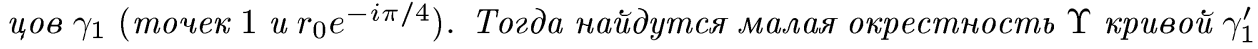
и числа $u_{0}>0, C>0$ такие, что при всех $и>u_{0}$ область $\Upsilon$ будет содержать точки спектра. Более того, если рассмотреть точки $\tilde{\lambda}_{k}$, определяемые уравнениями

$$
e^{\pi i / 4} J\left(\tilde{\lambda}_{k}\right)=\frac{i(\pi k-\pi / 4)}{u}
$$

то в кажддой окрестности $\mathscr{U}_{k} \subset \Upsilon$ найдутся ровно две точки спектра. При этом в $\Upsilon$ нет других точек спектра, не лежащих в $\mathscr{U}_{k}$.

ДокАЗАТЕЛЬСтво. Выберем окрестность $\Upsilon$ кривой $\gamma_{1}^{\prime}$ так, чтобы функция $J(\lambda)$ была однолистной в $\Upsilon$. Воспользовавшись матрищей перехода (5.7) и повторив рассуждения из леммы 5.7 , найдем, что равенство нулю характеристического определителя при $\lambda \in \Upsilon$ эквивалентно равенству

$$
\left(i e^{2 u e^{i \pi / 4} J(\lambda)}-1\right)^{2}=O\left(\frac{1}{u}\right)
$$

Применение теоремы Руше завершает доказательство леммы.

Наконец, определим поведение собственных значений в окрестности кривой $\gamma_{\infty}$.

ЛЕмма 5.12. Пусть $\Upsilon$ - область в $D=\{\lambda \in \Pi \mid \arg \lambda<-\pi / 4\}$, причем $\Upsilon$ содержит $\gamma_{\infty}$ c выколотой точкой-узлом $r_{0} e^{-i \pi / 4}$ (замыкание $\Upsilon$ не содержит $\left.r_{0} e^{-i \pi / 4}\right)$. Тогда $\Upsilon$ содержит точки спектра при больших $u>u_{0}$. Более того, если рассмотреть точки $\tilde{\lambda}_{k} \in \Upsilon$, определяемые уравнением $e^{i \pi / 4} K\left(\tilde{\lambda}_{k}\right)=i \pi k$, то существует число $C$ такое, что в каждой окрестности $\mathscr{U}_{k}$ этих точек радиуса $\mathrm{Cu}^{-2}$ найдется ровно одна точка спектра. Вне окрестностей $\mathscr{U}_{k}$ область $\Upsilon$ точек спектра не содержит.

ДоКАЗАТЕЛЬСтво. Равенство нулю характеристического определителя в этом случае эквивалентно равенству

$$
e^{2 u e^{i \pi / 4} K(\lambda)}=1+O\left(\frac{1}{u}\right)
$$

Корни невозмущенного уравнения определяются из равенства $2 u e^{i \pi / 4} K\left(\tilde{\lambda}_{k}\right)=$ $2 \pi k i$. Доказательство снова завершается применением теоремы Руше.

Следуюший результат является усилением теоремы 4.1.

ТЕОРема 5.1. Пусть $\gamma=\gamma_{0} \cup \gamma_{1} \cup \gamma_{\infty}$ - предельный спектральный граф оператора $L(\varepsilon)$. Для любого $\delta>0$ существуют постоянные $C>0, u_{0}>0$ такие, что все собственные значения оператора $L(\varepsilon)$ лежат при $u>u_{0} \boldsymbol{в}$ оббединении $\mathrm{Cu}^{-2}$-окрестности предельного графа $\gamma$ и $\delta$-окрестностей точек 0,1 и узла $r_{e}^{-i \pi / 4}$. При этом все собственные значения, лежащие вне 
$\delta$-окрестностей указанных точек, лежат в $\mathrm{Cu}^{-2}$-окрестностях точек $\tilde{\lambda}_{k}$, которые определяются соотношениями

$$
\tilde{\lambda}_{k}=(2 k+1) u^{-1} e^{-i \pi / 4}, \quad \tilde{\lambda} \in \gamma_{0},
$$

$\tilde{\lambda}_{k}-$ нули уравнения ие $e^{i \pi / 4} J(\lambda)=i(\pi k-\pi / 4), \quad \tilde{\lambda} \in \gamma_{1}, \quad \tilde{\lambda}_{k}-$ нули уравнения $u e^{i \pi / 4} K(\lambda)=\pi k i, \quad \tilde{\lambda} \in \gamma_{\infty}$.

Для $\tilde{\lambda}_{k} \in \gamma_{0} \cup \gamma_{\infty}$ окрестности $\mathscr{U}_{k}$ содержат по одному собственному значению, а для $\tilde{\lambda}_{k} \in \gamma_{1}-$ по два с учетом кратности.

ДоКАЗАТЕЛЬСтво. Этот результат есть следствие лемм 5.7, 5.10 и 5.11.

ОПРЕДЕЛЕНИЕ 5.4. Пусть $\gamma(t), t \in[0,1],-$ ориентированная гладкая кривая в плоскости $\mathbb{C}$ с начальной точкой $z_{0}$ и конечной $z_{1}$ (одна или обе точки могут принимать значения $\infty$ ). Запишем $\lambda_{1} \prec \lambda_{2}$, если $\lambda_{j}=\gamma\left(t_{j}\right)$ и $t_{1}<t_{2}$. Пусть $\Pi_{\delta}\left(\gamma, \lambda_{1}, \lambda_{2}\right)$ - криволинейная полоса шириной $2 \delta$, для которой $\gamma$ является средней линией, а боковыми сторонами служат отрезки, проходящие через $\lambda_{1}$ и $\lambda_{2}$ и перпендикулярные к $\gamma$. Пусть $F(z)$ голоморфна в $\Pi_{\delta}\left(\gamma, z_{0}, z_{1}\right)$. Фиксируем точку $\lambda_{1} \in \gamma$ и обозначим через $n\left(\lambda_{1}, \lambda\right)$ число нулей функции $F(z)$ в $\Pi_{\delta}\left(\gamma, \lambda_{1}, \lambda\right)$, если $\lambda \succ \lambda_{1}$. При $\lambda \prec \lambda_{1}$ полагаем $n\left(\lambda_{1}, \lambda\right)=-n\left(\lambda, \lambda_{1}\right)$. Функцию $N(\lambda)=n\left(\lambda_{1}, \lambda\right)+C$, где $C$-произвольная постоянная, назовем функцией распределения нулей $F(z)$ в $\delta$-окрестности кривой $\gamma$ (или вдоль кривой $\gamma$ ).

Так как собственные значения оператора $L(\varepsilon)$ являются нулями характеристического определителя $\Delta(u, \lambda)$, то аналогичное определение сохраняем для функций распределения собственных значений вдоль кривых.

Следуюшая теорема вместе с теоремой 5.1 представляют основной результат работы.

ТЕОРема 5.2. Функиии распределения собственных значений оператора $L(\varepsilon)$ вдоль кривых $\gamma_{\infty}, \gamma_{0}$ и $\gamma_{1}$ имеют следующий вид:

$$
\begin{aligned}
& N(\lambda)=\frac{u}{\pi} e^{-\frac{\pi}{4} i} \int_{-1}^{1} \sqrt{\zeta^{2}-\lambda} d \zeta+O(1) \quad \text { npu } \quad \lambda \in \gamma_{\infty}, \\
& N(\lambda)=\frac{u}{2} e^{\frac{\pi}{4} i} \lambda+O(1) \quad \text { npu } \quad \lambda \in \gamma_{0}, \\
& N(\lambda)=\frac{2 u}{\pi} e^{-\frac{\pi}{4} i} \int_{\sqrt{\lambda}}^{1} \sqrt{\zeta^{2}-\lambda} d \zeta+O(1) \quad \text { npu } \quad \lambda \in \gamma_{1} .
\end{aligned}
$$

В этих формулах остаток $O(1)$ оценивается константой, не зависящей от и и $\lambda$, если $\lambda$ находится вне фиксированных малых окрестностей концевых точек кривых.

ДокАЗАТЕЛЬСТво. Рассмотрим подробно лишь случай кривой $\gamma_{1}$, для других кривых доказательство аналогично.

Пусть точки $\lambda_{1}, \lambda \in \gamma_{1}$ не совпадают с концами $\gamma_{1}$. Рассмотрим полосу $\Pi_{\delta}\left(\gamma_{1}\right.$, $\left.\lambda_{1}, \lambda\right)$, о которой говорилось в определении 5.5. Отметим все точки $\tilde{\lambda}_{k}$, заданные соотношениями (5.8) и такие, что $\tilde{\lambda}_{k} \in \Pi_{\delta}\left(\gamma_{1}, \lambda_{1}, \lambda\right)$. Пусть $k_{\min }$ и $k_{\max }-$ минимальный и максимальный индексы отмеченных точек $\tilde{\lambda}_{k}$. Тогда число $N_{0}$ отмеченных точек равно $k_{\max }-k_{\min }$. Найдутся числа $u_{0}>0, C>0$ такие, что при 
$u>u_{0}$ окрестности $\mathscr{U}_{k}$ отмеченных точек $\tilde{\lambda}_{k}$ радиуса $C u^{-2}$ не пересекаются друг с другом, поэтому согласно теореме 5.1 при $u>u_{0}$ число собственных значений в $\Pi_{\delta}\left(\gamma_{1}, \lambda_{1}, \lambda\right)$ равно

$$
N\left(\gamma_{1}, \lambda_{1}, \lambda\right)=2 N_{0}+R
$$

где $0 \leqslant R \leqslant 4$. С другой стороны, из определения точек $\tilde{\lambda}_{k}$ легко получаем

$$
2 N_{0}=2 \frac{u}{\pi} e^{-i \pi / 4}\left(J\left(\tilde{\lambda}_{k_{\max }}\right)-J\left(\tilde{\lambda}_{k_{\min }}\right)\right)+R^{\prime}, \quad 0 \leqslant R^{\prime} \leqslant 4
$$

Так как расстояние между соседними точками $\tilde{\lambda}_{k}$ и $\tilde{\lambda}_{k+1}$ равно $\pi / u$, то

$$
\begin{aligned}
& \left|J\left(\lambda_{1}\right)-J\left(\tilde{\lambda}_{k_{\min }}\right)\right| \leqslant\left|J\left(\tilde{\lambda}_{k_{\min }-1}\right)-J\left(\tilde{\lambda}_{k_{\min }}\right)\right|=\frac{\pi}{u}, \\
& \left|J\left(\lambda_{2}\right)-J\left(\tilde{\lambda}_{k_{\max }}\right)\right| \leqslant\left|J\left(\tilde{\lambda}_{k_{\max }+1}\right)-J\left(\tilde{\lambda}_{k_{\max }}\right)\right|=\frac{\pi}{u} .
\end{aligned}
$$

Но тогда из (5.9) и (5.10) получаем

$$
\mathscr{N}\left(\gamma_{1}, \lambda_{1}, \lambda\right)=2\left(\frac{u}{\pi} e^{-\frac{\pi}{4} i}\left(J(\lambda)-J\left(\lambda_{1}\right)\right)+O(1)\right),
$$

что доказывает утверждение теоремы для кривой $\gamma_{1}$.

Авторы искренне благодарят М. И. Нейман-заде и А. М. Савчука за компютерную реализацию рисунков, имеюшихся в работе.

Результаты этой работы сообщались авторами на семинарах в МГУ в марте 2000 г., апреле 2001 г. и на конференции, посвященной 100-летию И. Г. Петровского, в мае 2001 г.

\section{Список литературы}

1. Шкаликов A.A. Асимптотическое поведение собственных значений одной модельной задачи // Матем. заметки. 1997. Т. 61. №6. С. 950-954.

2. Reddy S. G., Schmidt P.J., Henningson D.S. Pseudospectra of the Orr-Sommerfeld operator // SIAM J. Appl. Math. 1993. V. 53. № 1. P. 15-47.

3. Марченко М. А. Операторы Штурма-Лиувилля и их приложения. Киев: Наук. думка, 1977.

4. Наймарк М. А. Линейные дифференциальные операторы. М.: Наука, 1969.

5. Федорюк М. В. Асимптотические методы для линейных обыкновенных дифференциальных уравнений. М.: Наука, 1983.

6. Олвер Ф. Асимптотика и специальные функции. М.: Наука, 1990.

7. Tumanov S. N. Model problem for Poiseile profile. Critical spectrum curve // International Conference "Diff. Equations and related topics" dedicated to the Centenary Annyversary of I.G. Petrovskii. Book of Abstracts. M.: Moscow University, 2001. P. 413-414. 